\title{
EL HABLA JUVENIL DE SONORA, MÉXICO: LA FONÉTICA DE 32 JÓVENES
}

El habla de los jóvenes, parte del dialecto de una región, interesa porque muestra tendencias contrarias: por un lado la conformidad y por otro, la innovación. Los jóvenes tienden a conformarse con la lengua aprendida en el hogar en cuanto a la fonética, la morfología y el léxico. Al mismo tiempo, tienden a admitir conscientemente modales foráneos en un esfuerzo por adaptarse a su ambiente social.

Para estudiar la fonética del habla juvenil del estado de Sonora, México, se grabaron conversaciones con varios jóvenes. El tema del presente estudio es un análisis de los sonidos observados en el habla de 32 de esos jóvenes, 16 mujeres y 16 varones.

Se limitó la selección de informantes a (1) los que habían nacido en Sonora y (2) los que habían pasado toda su vida en el estado. Además se trató de conseguir (3) a personas cuya madre era sonorense también. Este último requisito, que se cumplió en todos los casos menos uno (el informante núm. 25), se debe a la hipótesis de que la lengua materna es formativa en el desarrollo del habla de los hijos.

Las edades de los informantes abarcaban desde los 16 hasta los 22 años. Del grupo de 32 informantes escogidos para este estudio, 30 eran estudiantes; otro era maestro de primaria y una informante trabajaba en un centro rural de salud. Para mantener cierto equilibrio en la selección de informantes, se hicieron grabaciones en dos escuelas. Trece fueron hechas en un colegio de internas, "Colegio San Francisco Javier", situado en San Ignacio, Distrito de Magdalena. Quince entrevistas se hicieron con alumnos y dos con alumnas en el "Instituto Tecnológico de Hermosillo", situado en la capital. Dos conversaciones fueron grabadas en Arizpe, Río Sonora.

Los informantes procedían de 19 comunidades de diferentes 
regiones del estado de Sonora. Del grupo, ocho habían nacido en un lugar distinto al pueblo o ciudad en donde vivían cuando se hizo la entrevista. Al contestar la pregunta: ¿De dónde es usted?, los informantes respondieron nombrando las siguientes localidades sonorenses (véase el mapa).

Informantes femeninas:

1. Granados

2. Puerto Peñasco (nacida en Magdalena)

3. Ciudad Obregón (nacida en Colonia Militar del Río Mayo)

4. San Pedro de la Cueva

5. San Ignacio (nacida en Huépac)

6. San Ignacio

Informantes masculinos:

17. Tepache

18. San Pedro de la Cueva

19. Bacanora (nacido en Sahuaripa)

20. Hermosillo

21. Rancho Viejo (nacido en Hermosillo)

22. Hermosillo

23. Cananea

24. Agua Prieta

25. Hermosillo (madre de Chihuahua)
7. San Ignacio

8. Hermosillo

9. San Pedro de la Cueva

10. Villa Juárez

11. Agua Prieta

12. Cócorit

13. La Galera

14. Hermosillo

15. Hermosillo

16. Arizpe
26. Hermosillo (nacido en Ciudad Obregón)

27. Hermosillo (nacido en Querobabi)

28. Campo Santa Elena

29. Magdalena de Kino

30. Hermosillo (nacido en El Saúz)

31. Santa Cruz

32. Ures

A los informantes se les preguntó acerca de la familia, el pueblo de donde venían, algunas celebraciones locales, los deportes, la vida estudiantil, los platos regionales, la música, etc. Los participantes tuvieron la oportunidad de escuchar la grabación después de hecha y de eliminar cualquier parte. Sólo en una ocasión tuvo que ser borrada una sección de una entrevista.

El plan de trabajo consistió en (1) hacer las grabaciones; (2) transcribirlas, pasando el texto a máquina; (3) escuchar las cintas 


\section{EL ESTADO DE SONORA, MEXICO}

con indicaciones del lugar de residencia y de nacimiento para los 32 informantes (1-16 femeninos, 17-32 masculinos)

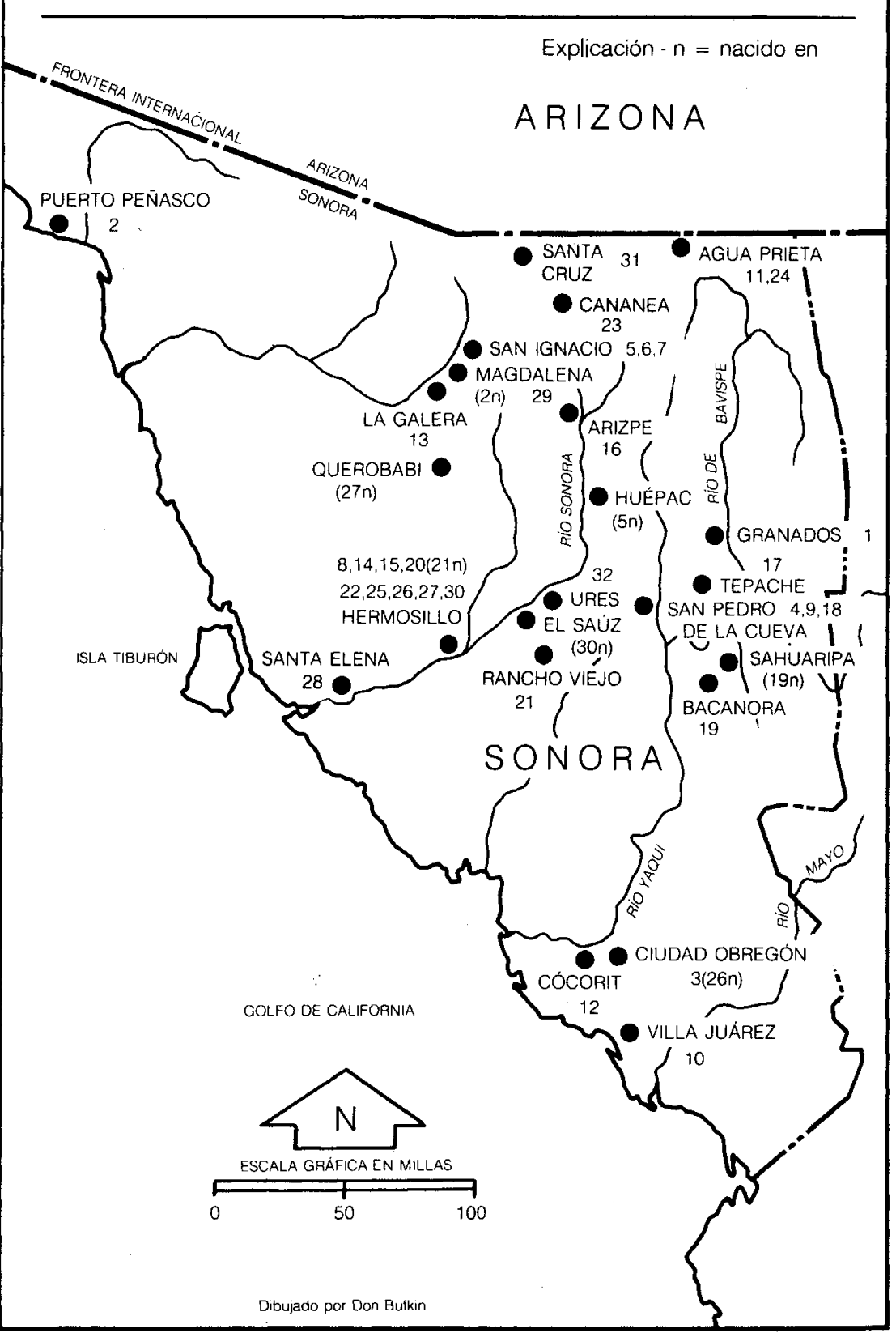


escribiendo el símbolo fonético para cada sonido analizado y (4) cuantificar la frecuencia de los distintos alófonos ${ }^{1}$.

Para dar una aproximación cuantitativa de la ocurrencia de las distintas variaciones alofónicas, se empleó una escala de cinco categorías: general, frecuente, medio, poco, esporádico. Este sistema para medir la frecuencia es una simplificación de la clasificación observada en el Atlas lingüístico de México dirigido por el Prof: Juan $\mathrm{M}$. Lope Blanch. No llega a la precisión matemática lograda en el Atlas porque no incluye los valores de "más" (+) y "menos" $(-)$.

General (G) describe el alófono usado casi exclusivamente en el ambiente señalado y constituye la norma para el informante.

Frecuente $(\mathrm{F})$ describe el alófono articulado más que ningún otro alófono en el ambiente indicado sin que sea de uso exclusivo.

Medio (M) describe el alófono que alterna con otro(s) alófono(s) en el mismo ambiente.

Poco (P) describe un alófono que se usa de vez en cuando pero no llega a ocurrir el $50 \%$ del tiempo.

Esporádico (E) describe el alófono que aparece en el habla de un informante, pero raras veces y sin poder establecer ningún patrón.

Los porcentajes dados indican el uso de un alófono y su frecuencia. Primero se dan los números para el grupo de los 32 informantes. Después se escriben los porcentajes para el grupo de 16 mujeres, y luego los porcentajes para los 16 informantes masculinos. El $100 \%$ para el grupo total consta de 32 personas. El $100 \%$ para los dos subgrupos consta de 16 informantes respectivamente ${ }^{2}$.

${ }^{1}$ Se empleó una grabadora marca "Sony", modelo TC-800B, Servocontrol. Las grabaciones se hicieron en cintas a la velocidad de $33 / 4 \mathrm{rpm}$. La ventaja de esa clase de grabadora es que le permite al investigador alargar o abreviar la velocidad de la grabación para poder concentrarse en algún detalle de la articulación.

${ }^{2}$ Tomando el caso de la $a$ tónica, uno encuentra que el $75 \%$ de los informantes estudiados, o sea, 24 personas, la pronuncian generalmente $(G)$ como vocal central baja oral [á]. De este grupo, más mujeres lo hacen así que hombres. El $93.75 \%$ de ellas, o sea 15 de 16 , tiene esta articulación mientras que sólo 9 de los 16 hombres (56.25\%) la pronuncian así generalmente. Tres informantes, núm. 1, la joven de Granados, núm. 21, el informante de Rancho Viejo nacido en Hermosillo y núm. 23, el joven de Cananea, pronuncian $a$ acentuada frecuentemente $(\mathrm{F})$ como vocal central baja. Dos informantes, núm. 24 y núm. 27, alternan [á] con un alófono velar [a] en uso medio (M).

Se notará que los informantes 1,21 y 23 pronuncian la $a$ acentuada algu- 


\section{VOCALES}

\section{Tónica central}

$\mathrm{La} / \mathrm{a} /$ tónica es articulada en general como vocal central baja oral por las tres cuartas partes de los jóvenes estudiados. Una variante velar [a] se encuentra en el habla de algunos de los varones; aparece relativamente poco en el habla de una mujer.

lal

\begin{tabular}{|c|c|c|c|c|c|c|c|c|c|c|c|c|c|}
\hline & & Todos & & & & & & & IF & & & $I M$ & \\
\hline [á] & G & 75.00 & & & & & & G & 93.75 & & G & 56.25 & \\
\hline & $\mathrm{F}$ & 9.375 & & & & & & $\mathrm{~F}$ & 6.25 & & $\mathrm{~F}$ & 12.50 & \\
\hline & M & 6.25 & & & & & & M & 0 & & M & 12.50 & \\
\hline & $P$ & 9.375 & & & & & & $\mathrm{P}$ & 0 & & $P$ & 18.75 & \\
\hline & E & 0 & & & & & & E & 0 & & E & 0 & \\
\hline [á] & G & 0 & & & & & & G & 0 & & G & 0 & \\
\hline & $\mathrm{F}$ & 9.375 & & & & & & $\mathrm{~F}$ & 0 & & $F$ & 18.75 & \\
\hline & M & 6.25 & & & & & & M & 0 & & M & 12.50 & \\
\hline & $P$ & 9.375 & & & & & & $\mathbf{P}$ & 6.25 & & $\mathrm{P}$ & 12.50 & \\
\hline & E & 0 & & & & & & E & 0 & & E & 0 & \\
\hline & G & 75.00 & & & & & [á] & G & 93.75 & [á] & G & 56.25 & \\
\hline [á] & $\mathrm{F}$ & 9.375 [á] & $P$ & 9.375 & [á] & P 6.25 & [á] & $\mathrm{F}$ & 6.25 & [á] & $\mathrm{F}$ & 12.50 [á] & $\begin{array}{ll}\mathrm{P} & 12.50\end{array}$ \\
\hline [a] [á] & M & 6.25 & & & & & & & 100.00 & [á] [á] & M & 12.50 & \\
\hline [a] & $\mathrm{F}$ & 9.375 [á] & $P$ & 9.375 & & & & & & [á] & $\mathrm{F}$ & 18.75 [á] & $\begin{array}{ll}\text { P } & 18.75\end{array}$ \\
\hline & & 100.00 & & & & & & & & & & 100.00 & \\
\hline
\end{tabular}

nas veces como sonido velar [a]. Es infrecuente tal articulación. El uso es poco (P). De manera que el uso frecuente $(F)$ de [a] se complementa con el poco uso (P) de [á]. No se incluye uso esporádico en el total de $100 \%$.

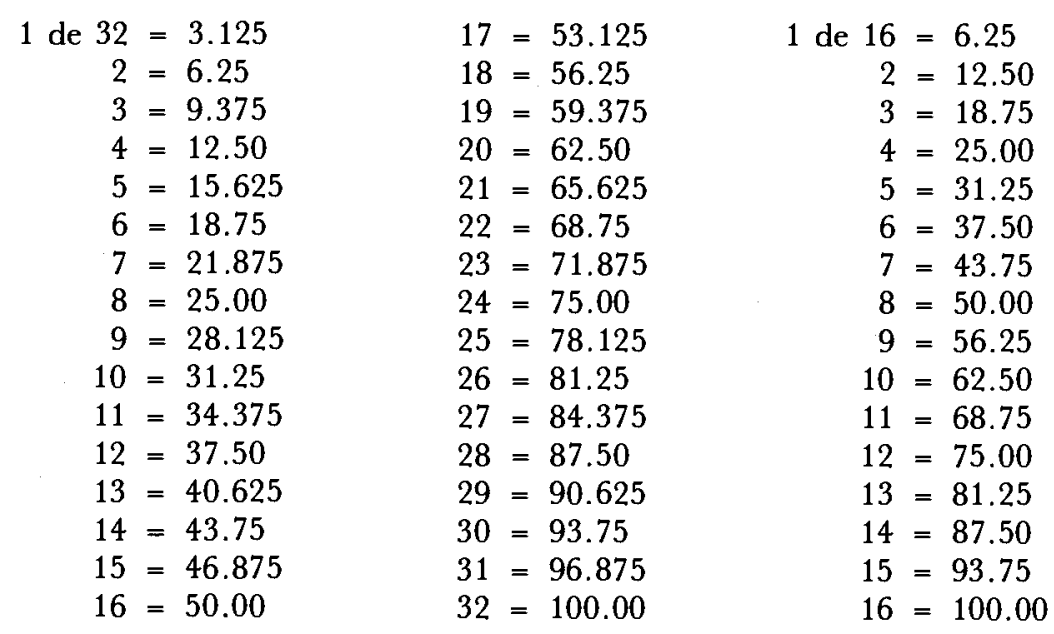




\section{Tónicas anteriores}

La /e/ generalmente es pronunciada como vocal media anterior oral [é] por la mayoría de los informantes jóvenes, con poca variación. Aunque la cerrazón a [é] no es general en la pronunciación de ninguno, sin embargo es observable en el habla de seis. La /é/ extremadamente cerrada [ é ] no se percibe en las grabaciones.

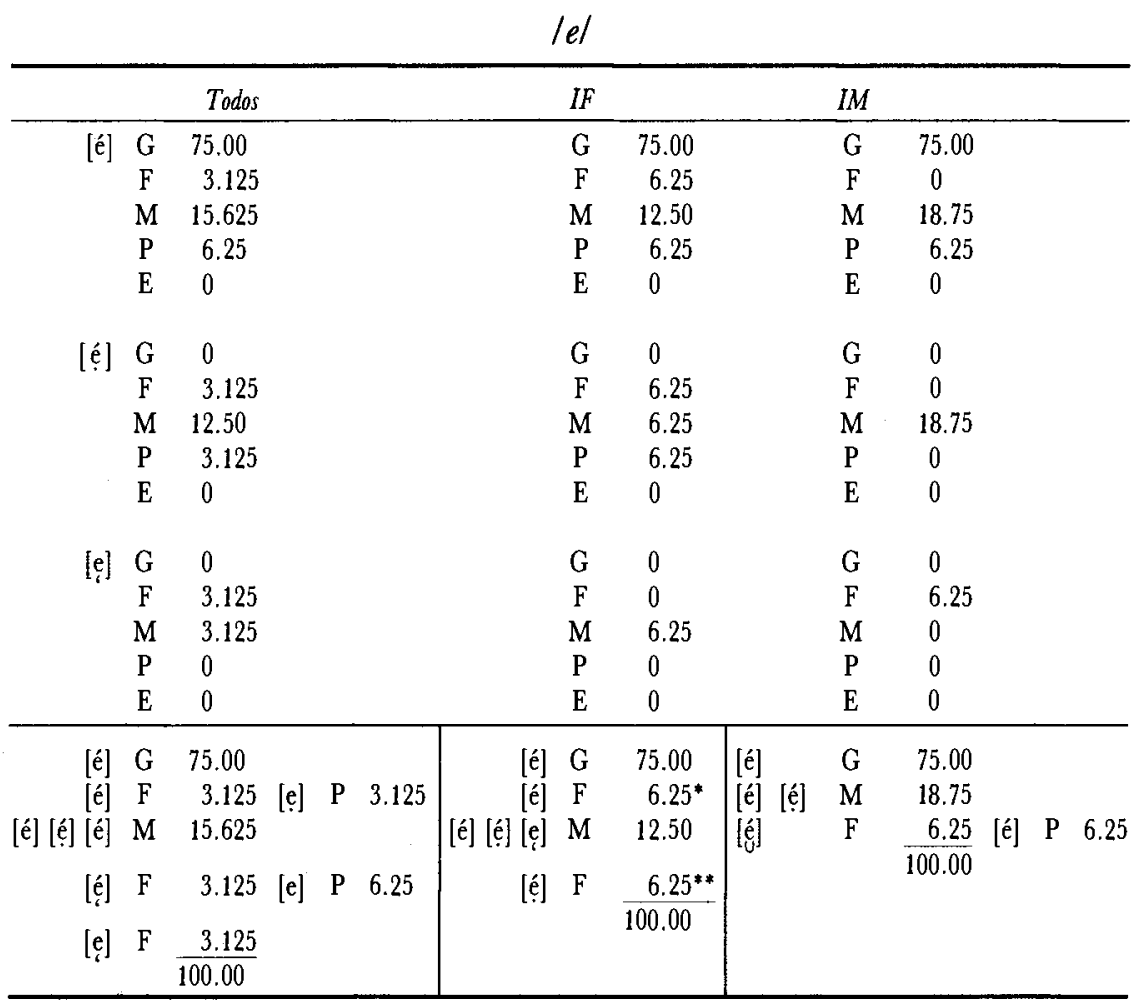

*[é] P 6.25

$* *[\mathrm{e}] \mathrm{P} 6.25$

El alófono característico de /i/ es [í], vocal alta anterior oral. Es la articulación generalmente observada entre los jóvenes. 
Tónicas posteriores

La característica más notable de la pronunciación de la vocal media posterior en posición tónica es la tendencia que tiene la generación joven sonorense hacia la elevación a [ọ́. La abertura a [ó] es normal en el habla de sólo una persona. La cerrazón extremada a [ọ́ ] no ha sido observada. [ọ́] ocurre como la articulación general en casi la tercera parte de los jóvenes y con frecuencia en otros tantos.

\section{$10 /$}

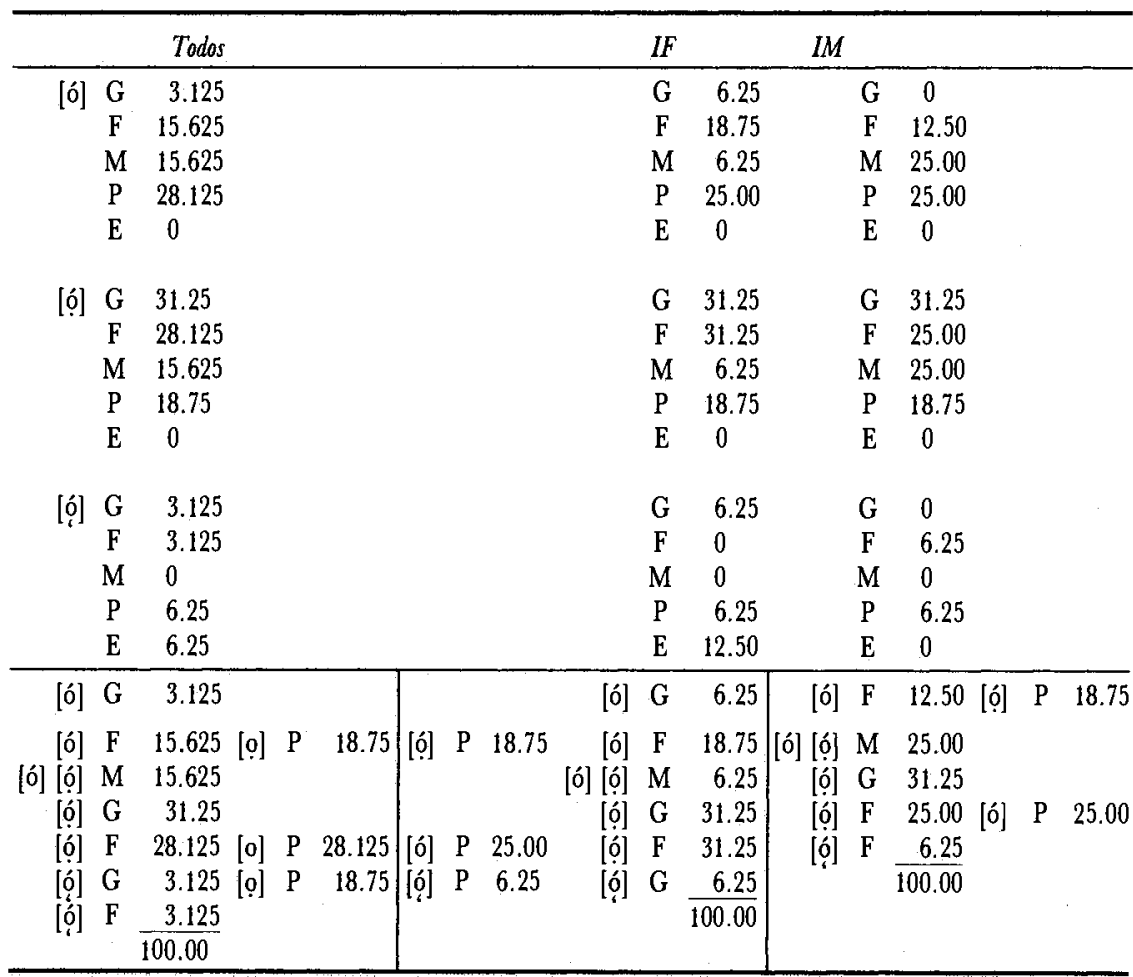

$\mathrm{La} / \mathrm{u} /$ se articula generalmente como [ú], vocal alta posterior oral. 
Átonas finales

Central. No se observan más variantes que el relajamiento en la pronunciación de $-\mathbf{a}[\mathrm{a}]$.

Anterior. La -e final átona es pronunciada [e] generalmente por más de las tres cuartas partes de los informantes. Tiende a cerrarse a [e] en el habla de unos pocos ( 7 de los 32). La [ e ] apenas se puede observar aunque ocurre en el habla de una persona con poca frecuencia.

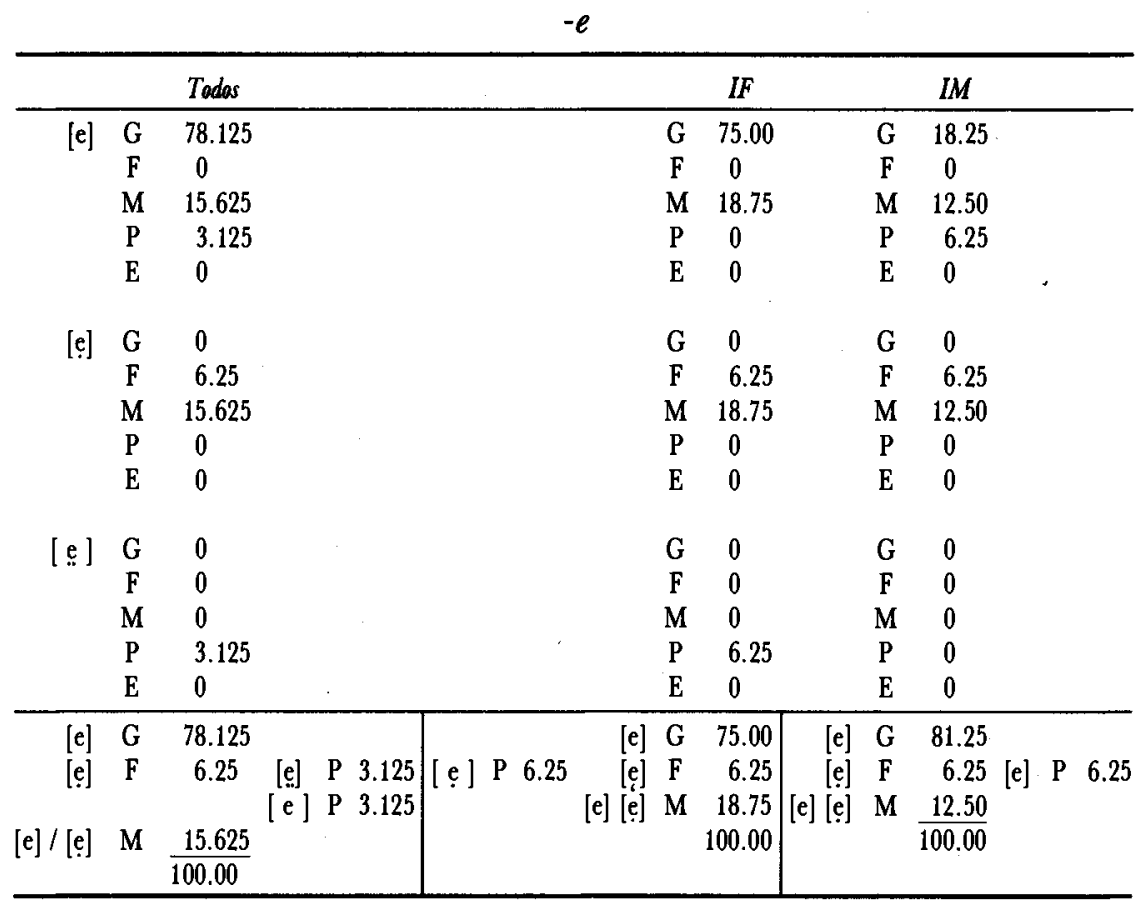


Posterior. En contraste con la [e] átona final, la elevación de -o final a [o] es general en el habla de 6 jóvenes y frecuente en la pronunciación de casi la mitad del grupo. Se puede considerar que el fenómeno de cierre a [o] constituye una variante característica del habla juvenil sonorense. Aunque el cierre a [ o ] no se generaliza en ninguno, no obstante, es un fenómeno que ocurre muy de vez en cuando en el habla de casi un tercio de los informantes estudiados (31.25).

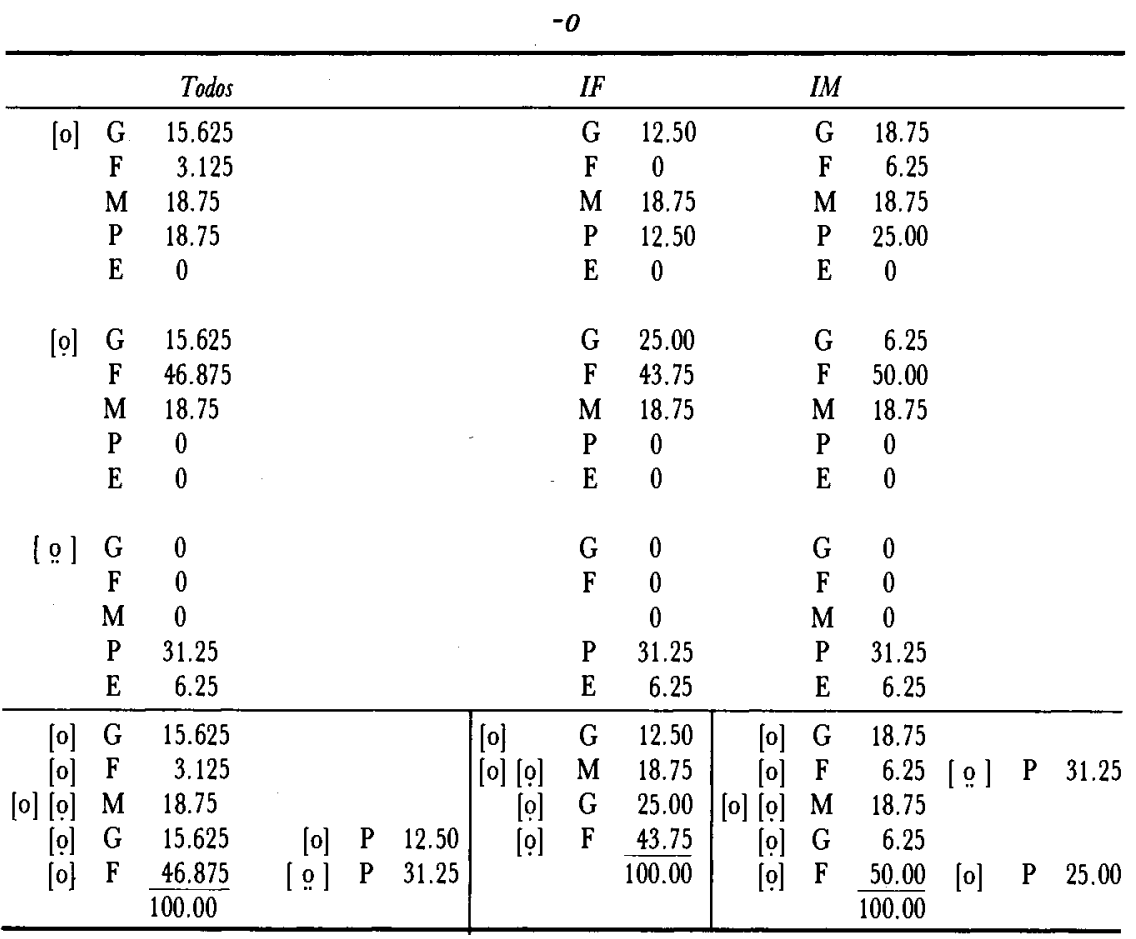




\section{Consonantes}

$|p|$

$\mathrm{La} / \mathrm{p} /$ se pronuncia [p] en el habla de todos los informantes masculinos y en el habla de $75 \%$ de las mujeres. Se nota como general la aspiración [p'] en una de las informantes femeninas y de poca frecuencia en otras. No es observable entre los hombres.

\begin{tabular}{|c|c|c|c|c|c|c|c|c|c|c|c|c|c|c|}
\hline & & Todos & & & & & & & & IF & & & $I M$ & \\
\hline \multirow[t]{5}{*}[\mathrm{p}]{} & G & 84.375 & & & & & & & & G & 75.00 & & $G$ & 100.00 \\
\hline & F & 9.375 & & & & & & & & $\mathrm{~F}$ & 18.75 & & $\mathbf{F}$ & 0 \\
\hline & M & 0 & & & & & & & & M & 0 & & M & 0 \\
\hline & $\mathrm{P}$ & 0 & & & & & & & & $P$ & 0 & & $P$ & 0 \\
\hline & E & 0 & & & & & & & & E & 0 & & E & 0 \\
\hline \multirow[t]{5}{*}[p^{\prime}]{} & G & 3.125 & & & & & & & & G & 6.25 & & $\mathrm{G}$ & 0 \\
\hline & $\mathrm{F}$ & 0 & & & & & & & & $\mathrm{~F}$ & 0 & & $\mathbf{F}$ & 0 \\
\hline & M & 0 & & & & & & & & M & 0 & & M & 0 \\
\hline & $P$ & 9.375 & & & & & & & & $P$ & 18.75 & & $P$ & 0 \\
\hline & $\mathrm{E}$ & 0 & & & & & & & & $\mathrm{E}$ & 0 & & E & 0 \\
\hline \multirow{4}{*}{$\begin{array}{l}{[\mathrm{p}]} \\
{[\mathrm{p}]} \\
{\left[\mathrm{p}^{\prime}\right]}\end{array}$} & G & 84.375 & & & & & & & {$[\mathrm{P}]$} & G & 75.00 & {$[\mathrm{P}]$} & G & 100.00 \\
\hline & $\mathrm{F}$ & 9.375 & {$\left[\mathrm{p}^{\prime}\right]$} & P & 9.375 & {$\left[\mathrm{p}^{\prime}\right]$} & $P$ & 18.75 & {$[\mathrm{p}]$} & $\mathrm{F}$ & 18.75 & & & \\
\hline & G & 3.125 & & & & & & & {$\left[\mathrm{p}^{\prime}\right]$} & G & $\begin{array}{l}6.25 \\
\end{array}$ & & & \\
\hline & & 100.00 & & & & & & & & & $\overline{100.00}$ & & & \\
\hline
\end{tabular}

$|t|$

La / $t$ / es articulada [t] por los 32 informantes estudiados. No se ha notado variante significativa. 


\section{$|k|$}

$\mathrm{La} / \mathrm{k} /$ es pronunciada generalmente como sonido velar oclusivo sordo [k] por el $81 \%$ de los informantes jóvenes, con la proporción de hombres más alta que la de mujeres $(93 \%$ a $68 \%)$. Una variante observada, pero de poca frecuencia, es la articulación del oclusivo con la lengua baja y el grado de oclusión notablemente reducido $[\mathrm{k} \perp]$. Aparece en el habla femenina, al igual que una leve aspiración [k'].

\section{$|k|$}

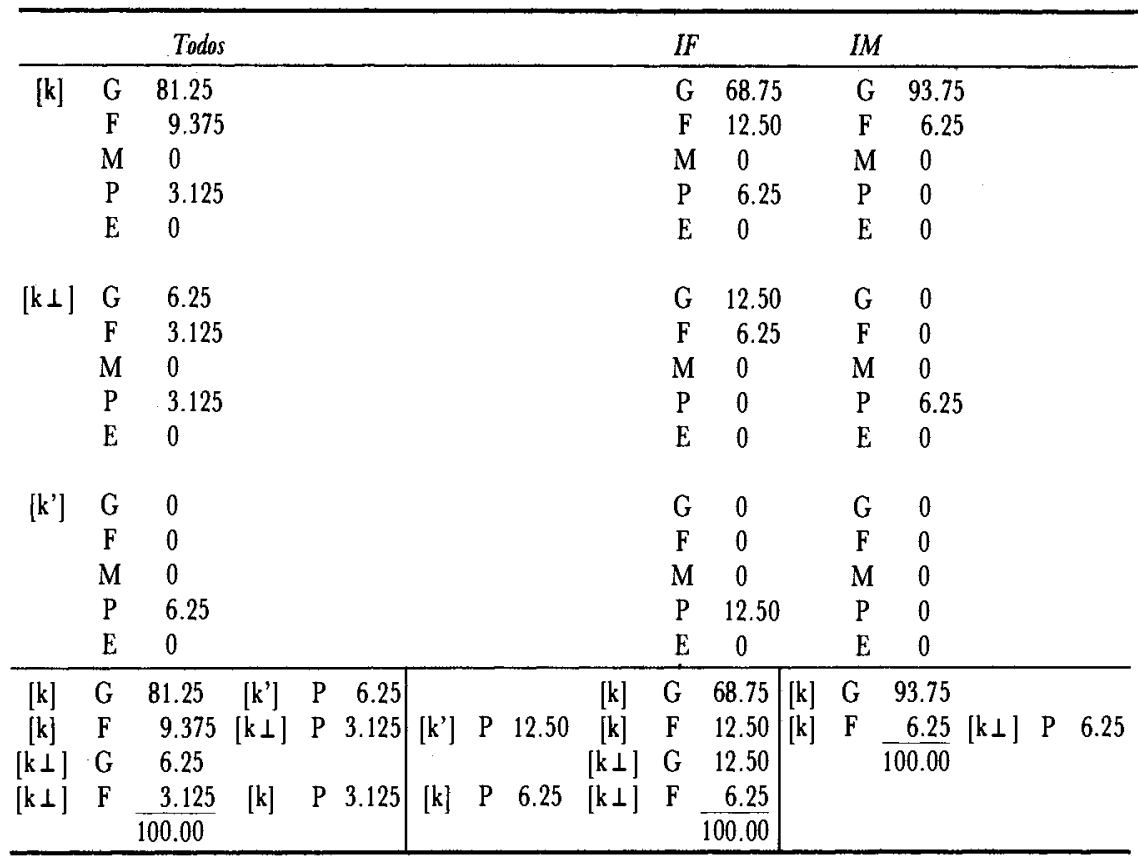


$|b|$

$b-$. La consonante $/ \mathrm{b} /$ inicial después de una pausa es articulada generalmente [b], bilabial oclusiva sonora.

Nasal $+b$. Después de un sonido nasal $/ \mathrm{b} /$ se articula [b] en el habla joven sonorense.

$-b-$. La /b/ en posición media es pronunciada $[b]$ por muchos informantes tanto regular como frecuentemente. Se puede observar oclusión en el habla de unos pocos [b] y el relajamiento a [ ${ }^{\mathbf{b}}$ ] en otros. Es decir, [b] y $\left[^{b}\right]$ se alternan en el habla de la mayoría de los jóvenes sonorenses con preferencia notada por [-b-].

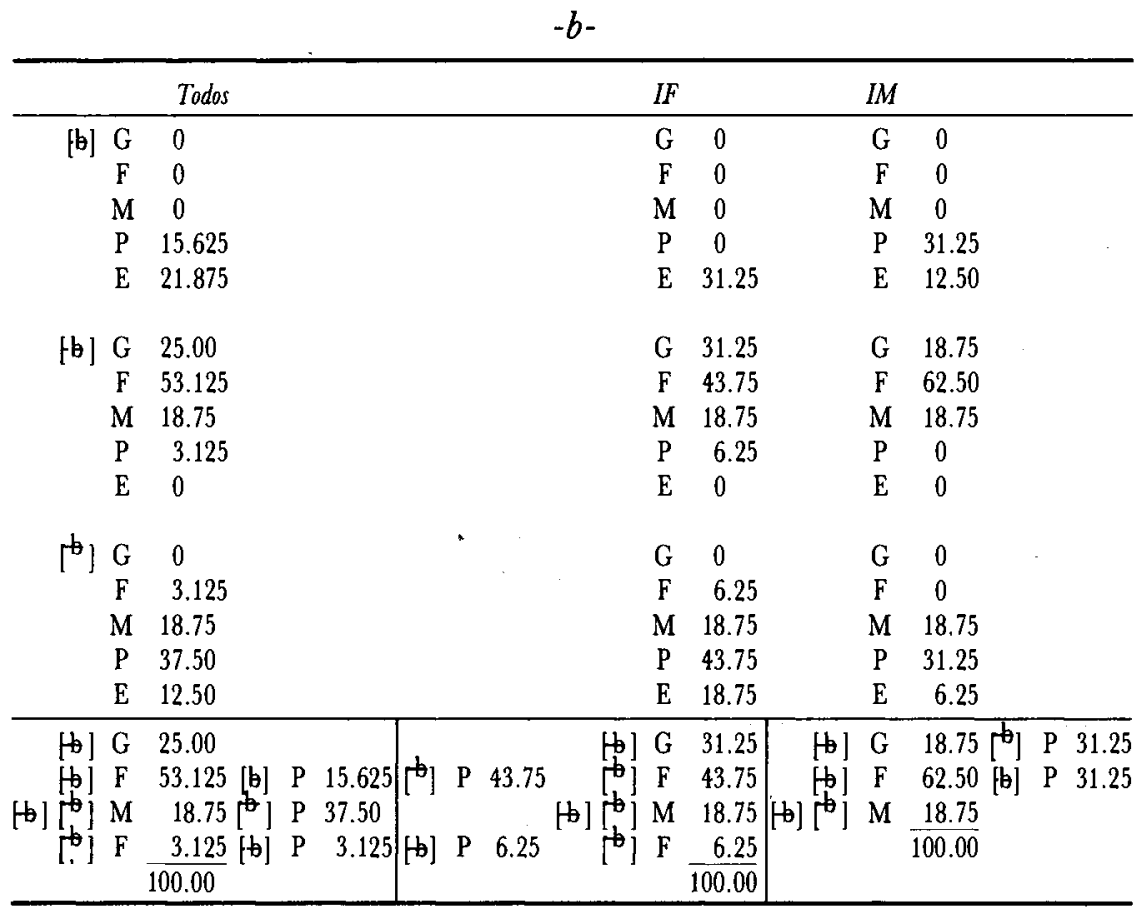


$/ d /$

$d$-. Inicialmente y después de una pausa, el alófono [d] ocurre generalmente en el habla de los sonorenses estudiados.

Nasal $+d$. Después de un nasal /d/ se pronuncia generalmente [d].

- $d$-. En el caso de - d- intervocálica, la articulación fricativa [-d- ] es la más frecuente entre los jóvenes sonorenses. También se observa la articulación relajada [ ] . La variante oclusiva [d] aparece esporádicamente en el uso de ambos sexos, aunque más entre las mujeres (56\% a $25 \%)$.

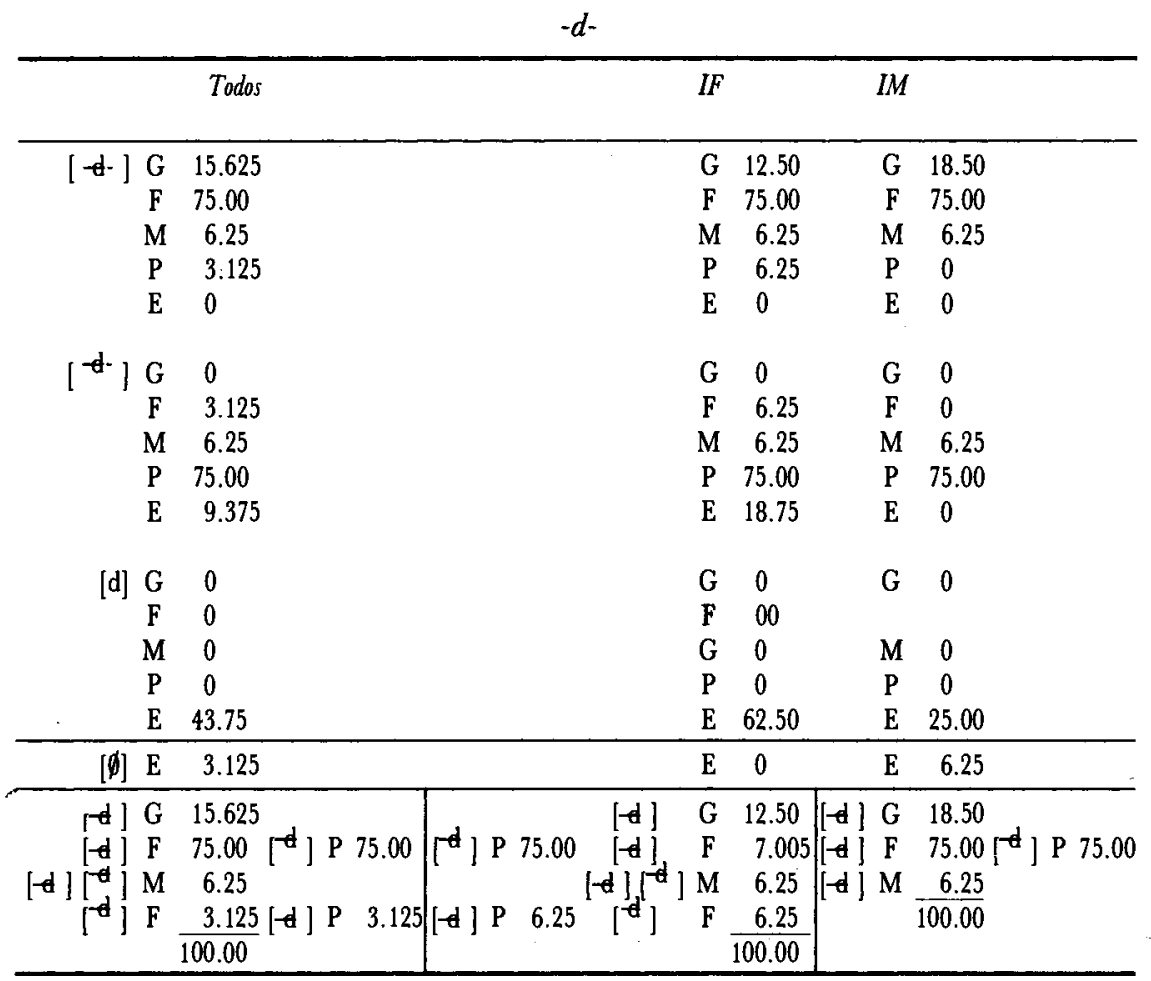


La palabra todo (-a, -os, -as) llama la atención por la frecuencia de relajamiento que ocurre en la $-\mathrm{d}-$ entre vocales. Según el análisis de las grabaciones hechas, hay una correlación entre la pronunciación relajada o fuerte y el sexo del informante. El 50\% de las mujeres pronuncian las formas de todo con ${ }^{+}$] generalmente, mientras que no se observa tal articulación relajada como uso general entre los varones.

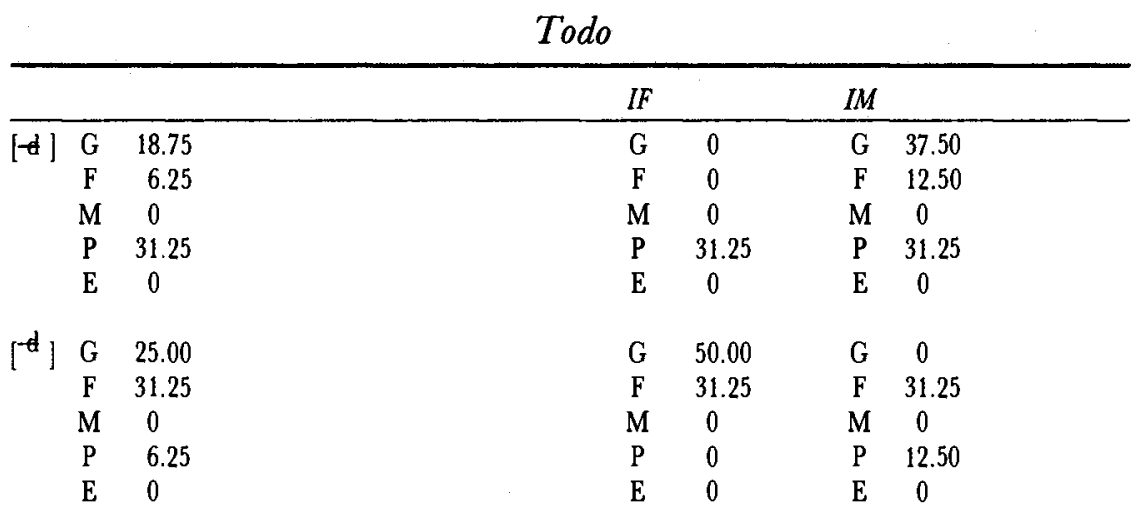

No aparece la palabra todo: $18.75 ;$ F 18.75 , M 18.75

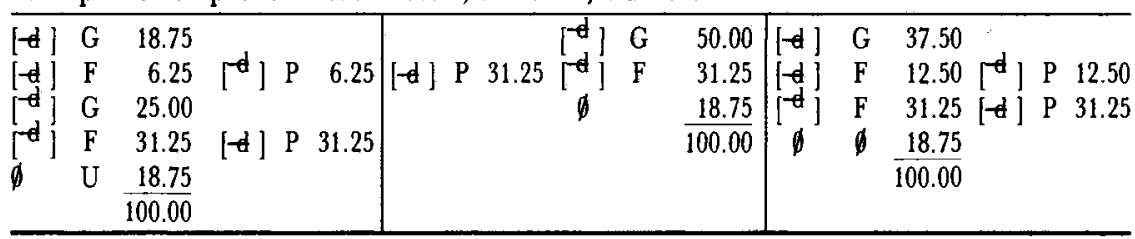

$|g|$

$g^{-}$. Los 14 informantes cuyas entrevistas contienen ejemplos de $\mathrm{g}$ - inicial después de pausa la pronuncian como velar oclusiva sonora $[g]$.

$g-$

\begin{tabular}{lllllll}
\hline & & & & $I F$ & $I M$ \\
\hline$[\mathrm{g}]$ & $\mathrm{G}$ & 53.125 & $\mathrm{G}$ & 62.50 & $\mathrm{G}$ & 43.75 \\
& No ocurre: & 46.857 & $\emptyset$ & 37.50 & $\emptyset$ & 56.25 \\
\hline
\end{tabular}


$N a s a l+g$. La articulación oclusiva es general en los 23 informantes que pronunciaron la combinación de nasal $+\mathrm{g}$ durante las conversaciones grabadas.

\begin{tabular}{llllllc}
\multicolumn{8}{c}{ nasal $+g$} \\
\hline [g] & G & 71.875 & G & 75 & G & IM \\
\hline & No ocurre: & 28.125 & $\emptyset$ & 25.00 & $\emptyset$ & 31.25 \\
\hline
\end{tabular}

-g- En posición media, el velar fricativo sonoro [-g- ] es el sonido más frecuente entre los jóvenes que constituyen este análisis.

También observable, aunque con frecuencia reducida, es la articulación relajada en que el dorso de la lengua no hace contacto firme con el velo del paladar [ $]$.

\begin{tabular}{|c|c|c|c|c|c|c|c|c|c|c|c|c|}
\hline & & Todos & & & & & & & IF & & $I M$ & \\
\hline \multirow[t]{5}{*}[-\mathbb{8}]{} & G & 56.25 & & & & & & G & 56.25 & G & 56.25 & \\
\hline & $\mathrm{F}$ & 43.75 & & & & & & $\mathrm{~F}$ & 43.75 & $\mathrm{~F}$ & 43.75 & \\
\hline & M & 0 & & & & & & M & 0 & $\mathrm{M}$ & 0 & \\
\hline & $\mathrm{P}$ & 0 & & & & & & $\mathbf{P}$ & 0 & $P$ & 0 & \\
\hline & $\mathrm{E}$ & 6.25 & & & & & & E & 12.50 & E & 0 & \\
\hline \multirow[t]{5}{*}[\begin{array}{l}{\mathrm{E}}\\
{\mathrm{B}}\end{array}]{} & G & 0 & & & & & & G & 0 & G & 0 & \\
\hline & $\mathrm{F}$ & 0 & & & & & & $\mathbf{F}$ & 0 & $\mathrm{~F}$ & 0 & \\
\hline & M & 0 & & & & & & M & 0 & M & 0 & \\
\hline & $\mathbf{P}$ & 37.50 & & & & & & $P$ & 37.50 & $\mathbf{P}$ & 37.50 & \\
\hline & E & 6.25 & & & & & & E & 12.50 & $\mathbf{E}$ & 0 & \\
\hline \multirow[t]{5}{*}{ [g] } & G & 0 & & & & & & G & 0 & G & 0 & \\
\hline & $\mathbf{F}$ & 0 & & & & & & F & 0 & F & 0 & \\
\hline & M & 0 & & & & & & M & 0 & M & 0 & \\
\hline & $\mathrm{P}$ & 6.25 & & & & & & $P$ & 6.25 & $\mathbf{P}$ & 6.25 & \\
\hline & E & 0 & & & & & & E & 0 & E & 0 & \\
\hline \multirow{3}{*}[\begin{array}{l}{-8}\\
{-3}\\
{-3}\end{array}]{} & G. & 56.25 & & & & & {$[-\vec{s}]$} & G & 56.25 & G & 56.25 & \\
\hline & $\mathrm{F}$ & 43.75 & [g] & $\mathrm{P}$ & 37.50 & & $\begin{array}{lll}\text { P } & 37.50[-5]\end{array}$ & $\mathrm{F}$ & 43.75 & $\mathbf{F}$ & 43.75 & 37.50 \\
\hline & & 100.00 & [g] & $P$ & 6.25 & {$[\mathrm{~g}]$} & $\begin{array}{ll}P & 6.25\end{array}$ & & 100.00 & & 100.00 & 6.25 \\
\hline
\end{tabular}


$g r$. La $g$ seguida por $r$ se articula más como sonido oclusivo (28\%) que como fricativo (18\%) en las conversaciones analizadas. No todos los informantes articularon la combinación consonántica $g r$ $(40 \%)$, pero en las grabaciones que la contienen, predomina $[\mathrm{g}]$.

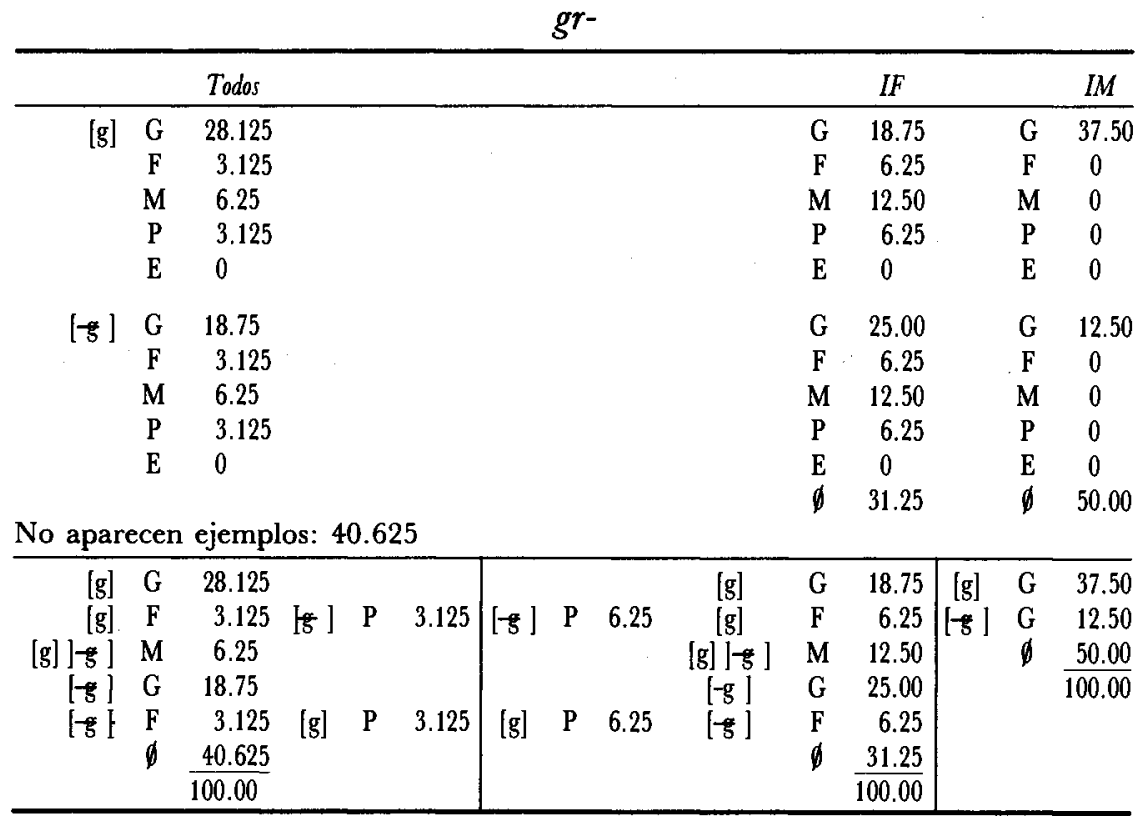

$|f|$

El sonido labiodental fricativo sordo [f] ocurre generalmente en el habla de los 32 informantes estudiados. No se han notado variantes alofónicas.

$|s|$

La pronunciación de los alófonos del fonema /s/ por parte de los jóvenes sonorenses revela diferencias significativas entre ambos sexos en cuanto al lugar y la manera de articularse. Esta observación corrobora la importancia de las variantes de /s/ en la determinación de dialectos y subdialectos de la lengua española.

$s-$. Los dos alófonos principales de s inicial son [s] consonante alveolar fricativa sorda y [s, consonante dental fricativa sorda.

Cuatro de los informantes masculinos pronuncian /s/generalmente de otra forma: dos con la lengua plana $[\overline{\mathrm{s}}]$ y dos con la lengua levemente cóncava [ $\mathrm{s}$ ] 
Se puede observar en el habla de tres informantes femeninas algún rasgo de articulación interdental $[\theta]$, sin que sea ésta de uso frecuente ni medio.

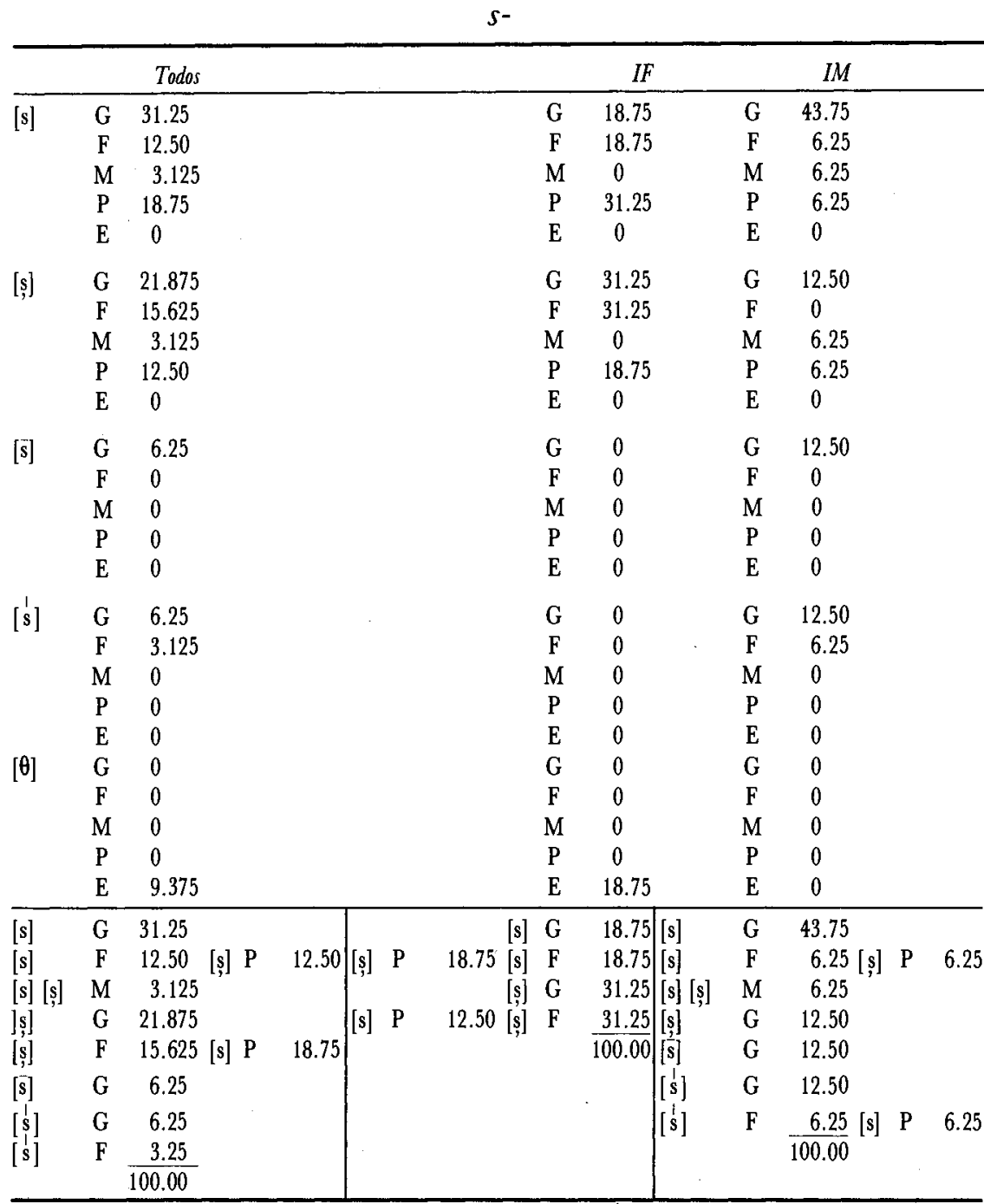

$-s-$. Los jóvenes sonorenses emplean mayormente [s] y [s] para $s$ en posición intervocálica. Se encuentran también otros alófonos $([\bar{s}],[\mathrm{z}],[\mathrm{s}],[\mathrm{h}],[\theta])$, pero con escasa frecuencia.

Aparecen diferencias de uso según el sexo del informante. Las jóvenes articulan el sonido como dental [ș] más que los jóvenes, para quienes el sonido más observado es el alveolar [s]. 


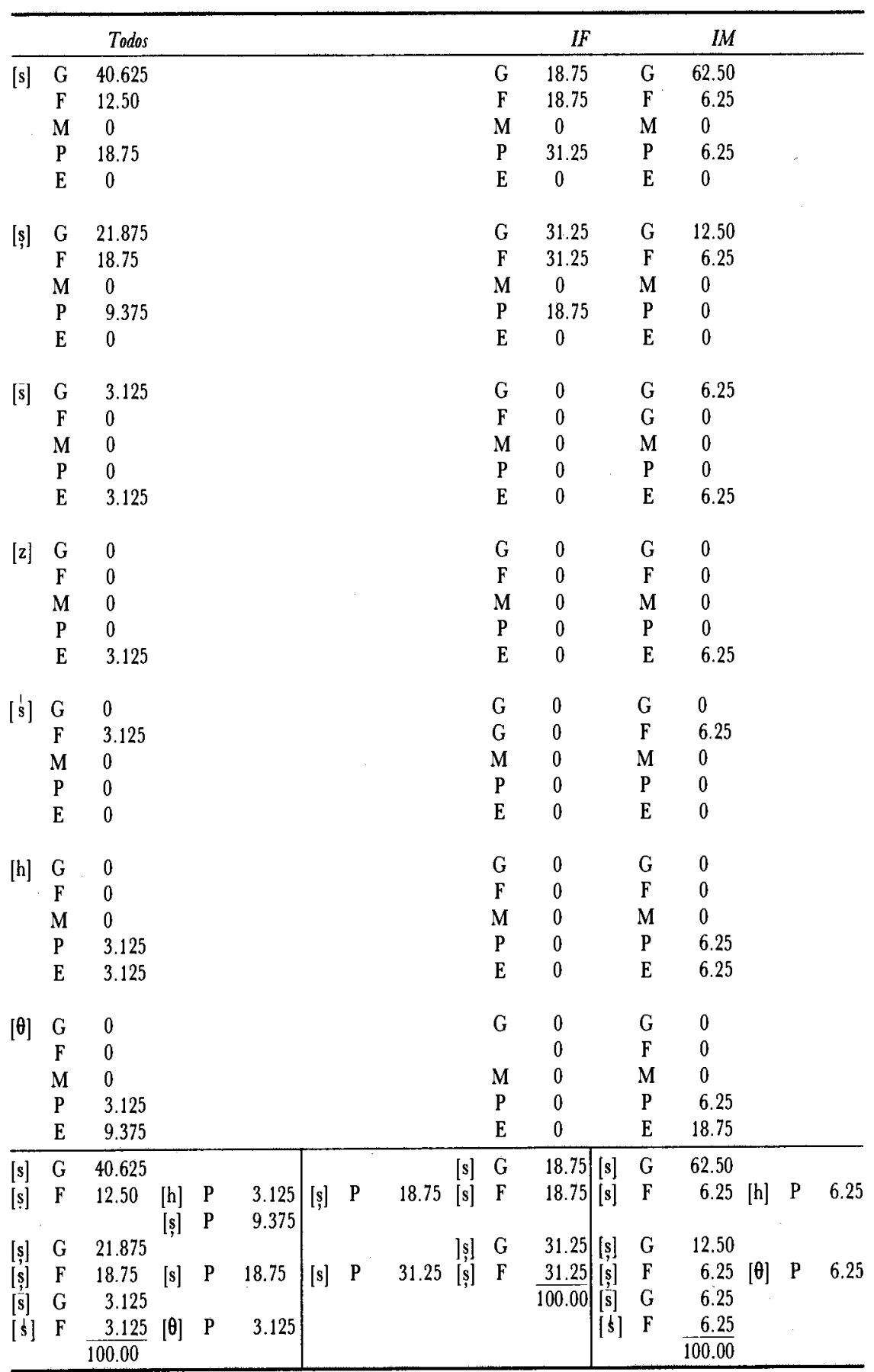


si. El hecho de que se palatalice o no la combinación de $s+$ yod + vocal es significante para diferenciar uso según el sexo del informante sonorense. Resulta que los varones tienden a palatalizar si mucho más que las mujeres. El $75 \%$ de aquéllos lo hace generalmente, comparado con el $12 \%$ de las mujeres. $\mathrm{Al}$ mismo tiempo, un aspecto del habla femenil merece comentario especial: en general, si evoca la articulación alveolar más que la dental (5 mujeres a 2). Hay menos distribución libre entre [s] y [ș] cuando $s$ es parte de la combinación $s i+$ vocal de la que existe cuando $s$ es inicial o intervocálica.

\begin{tabular}{ccccccc}
$s i$ \\
\hline$[s] o[s]$ sin palatalización \\
\hline Todos & & & & & \\
\hline $\mathrm{G}$ & 43.75 & $\mathrm{G}$ & 68.75 & $\mathrm{G}$ & 18.75 \\
\hline $\mathrm{F}$ & 0 & $\mathrm{~F}$ & 0 & $\mathrm{~F}$ & 0 \\
$\mathrm{M}$ & 3.12 & $\mathrm{M}$ & 6.25 & $\mathrm{M}$ & 0 \\
$\mathrm{P}$ & 0 & $\mathrm{P}$ & 0 & $\mathrm{P}$ & 0 \\
$\mathrm{E}$ & 0 & $\mathrm{E}$ & 0 & $\mathrm{E}$ & 0
\end{tabular}

$[s]$ con palatalización

\begin{tabular}{|c|c|c|c|c|c|c|c|}
\hline & & $\begin{array}{c}\mathrm{G} \\
\mathrm{F} \\
\mathrm{M} \\
\mathrm{P} \\
\mathrm{E}\end{array}$ & $\begin{array}{l}43.75 \\
0 \\
0 \\
0 \\
3.125\end{array}$ & $\begin{array}{c}\mathrm{G} \\
\mathrm{F} \\
\mathrm{M} \\
\mathrm{P} \\
\mathrm{E}\end{array}$ & $\begin{array}{l}12.50 \\
0 \\
0 \\
0 \\
6.25\end{array}$ & $\begin{array}{c}\mathrm{G} \\
\mathrm{F} \\
\mathrm{M} \\
\mathrm{P} \\
\mathrm{E}\end{array}$ & $\begin{array}{c}75.00 \\
0 \\
0 \\
0 \\
0\end{array}$ \\
\hline & {$[\theta]$} & $\begin{array}{c}G \\
F \\
M \\
P \\
E\end{array}$ & $\begin{array}{l}3.125 \\
0 \\
3.125 \\
0 \\
0\end{array}$ & $\begin{array}{c}\mathrm{G} \\
\mathrm{F} \\
\mathrm{M} \\
\mathrm{P} \\
\mathrm{E}\end{array}$ & $\begin{array}{l}6.25 \\
0 \\
6.25 \\
0 \\
0\end{array}$ & $\begin{array}{c}\mathrm{G} \\
\mathrm{F} \\
\mathrm{M} \\
\mathrm{P} \\
\mathrm{E}\end{array}$ & $\begin{array}{l}0 \\
0 \\
0 \\
0 \\
0\end{array}$ \\
\hline Sin ejemplos de si : 6.25 & & & & & 6.25 & & 6.25 \\
\hline $\begin{array}{l}{[\mathrm{s}][\mathrm{s}] \text { sin palatalización }} \\
{[\overline{\mathrm{s}}][\mathrm{s}] \text { sin palatalización }}\end{array}$ & {$[\theta]$} & $\begin{array}{l}G \\
G\end{array}$ & $\begin{array}{c}43.75 \\
3.125\end{array}$ & $\begin{array}{l}\mathrm{G} \\
\mathrm{M}\end{array}$ & $\begin{array}{r}68.75 \\
6.25\end{array}$ & G & 18.75 \\
\hline$[\stackrel{\vee}{s}$ ] con palatalización & {$[\theta]$} & G & 43.75 & G & 12.50 & G & 75.00 \\
\hline Sin ejemplos de $s \underset{n}{\mathrm{i}}$ & & G & $\begin{array}{r}3.125 \\
6.25 \\
100.00 \\
\end{array}$ & G & $\begin{array}{r}6.25 \\
6.25 \\
100.00 \\
\end{array}$ & & $\begin{array}{r}6.25 \\
100.00 \\
\end{array}$ \\
\hline
\end{tabular}


-s. En el habla juvenil sonorense, se encuentran seis alófonos para -s final de sílaba y final de sílaba más consonante sorda: [s], $[\mathrm{s}],[\bar{s}],[\mathrm{s}],[\mathrm{h}]$ y $[\theta]$. La mayoría de los casos de esta clase de $s$ final contienen o el sibilante alveolar [s] o el dental [ș]. Tal observación coincide con la situación cuando $s$ es inicial e intervocálica.

Conviene señalar que casi las dos terceras partes de los informantes masculinos articulan la $-s$ final como alveolar general y frecuentemente. Esta estadística contrasta con el uso femenino, el cual muestra la articulación alveolar y la dental en proporciones parecidas $(25 \%)$.

La omisión de -s es rara en la pronunciación de los 32 jóvenes analizados; $s$ final de sílaba y $s$ final seguida de consonante sorda son regularmente articuladas.

Varios lingüistas han identificado el vigor de la consonante como rasgo del dialecto mexicano ${ }^{3}$. La articulación persistente de /s/ en el habla juvenil sonorense refuerza esa aserción.

3 “. . . el habla popular del Valle de México se caracteriza por su fuerte consonantismo" (JOSEPH MATLUCK, "La pronunciación del español en el Valle de México", NRFH, 6 (1952), p. 111. "Among other speakers of Spanish, a Mexican is recognized. . . by his tendency to lengthen the articulation of $/ \mathrm{s} /$, and by a general preference for the consonant over the vowel" (D. LincolN CANFIELD, Spanish ipronunciation in the Americas, University of Chicago Press, Chicago, 1981, p. 60). 
-s final de silaba y -s final + consonante sorda

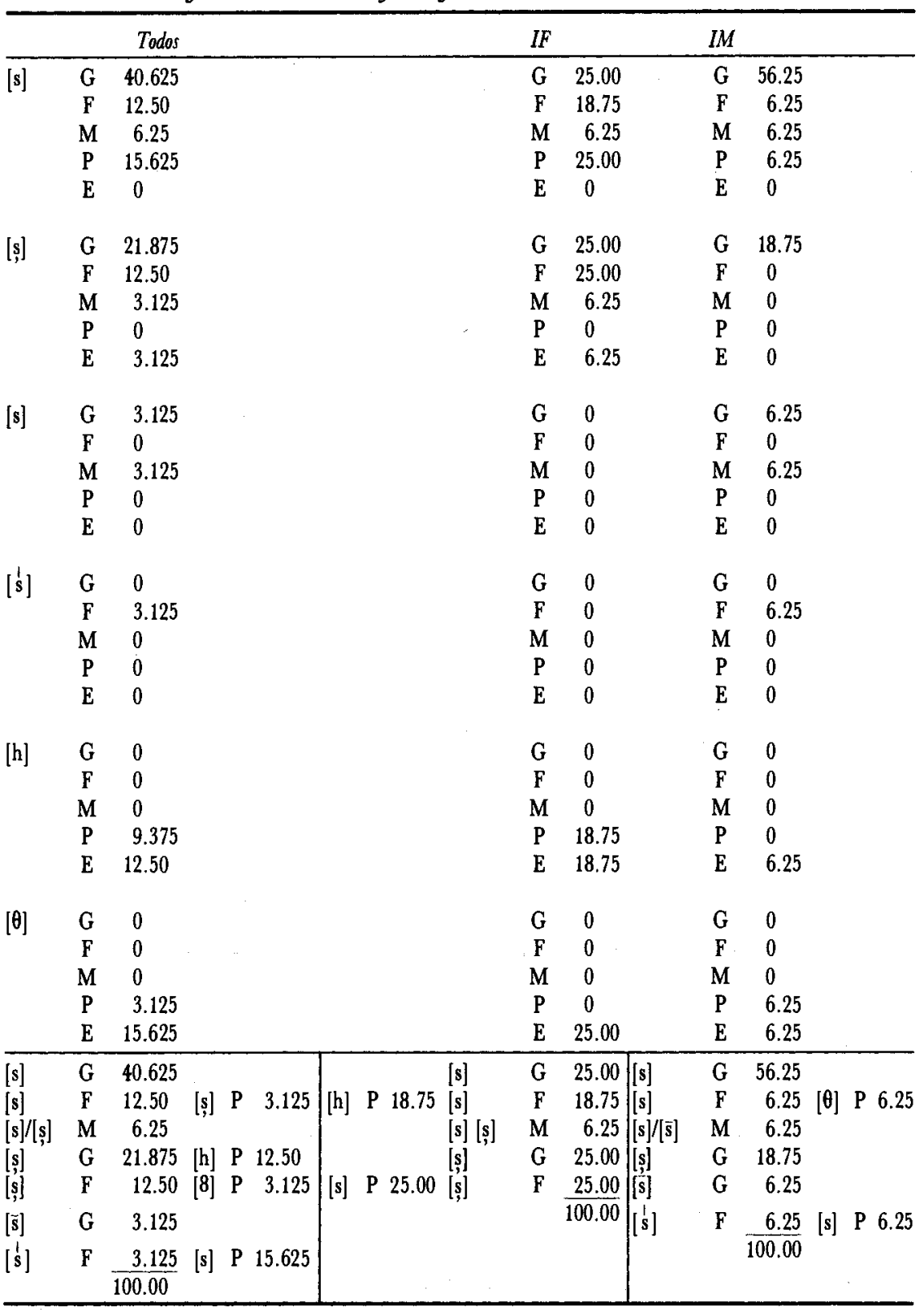


$\mathrm{El}$ ambiente de $-s$ final de sílaba + consonante sonora evoca tres alófonos, dos de ellos principales, [s] y [s], y uno de frecuencia más limitada, [h]. También se dan variantes relajadas de los dos primeros $\left[{ }^{\mathrm{S}}\right]$ y $[\mathrm{z}]$, las cuales se incluyen en [s] y [z]. La falta de regularidad en el uso de un alófono u otro indica la variación libre que existe en la articulación de $-s+$ consonante sonora.

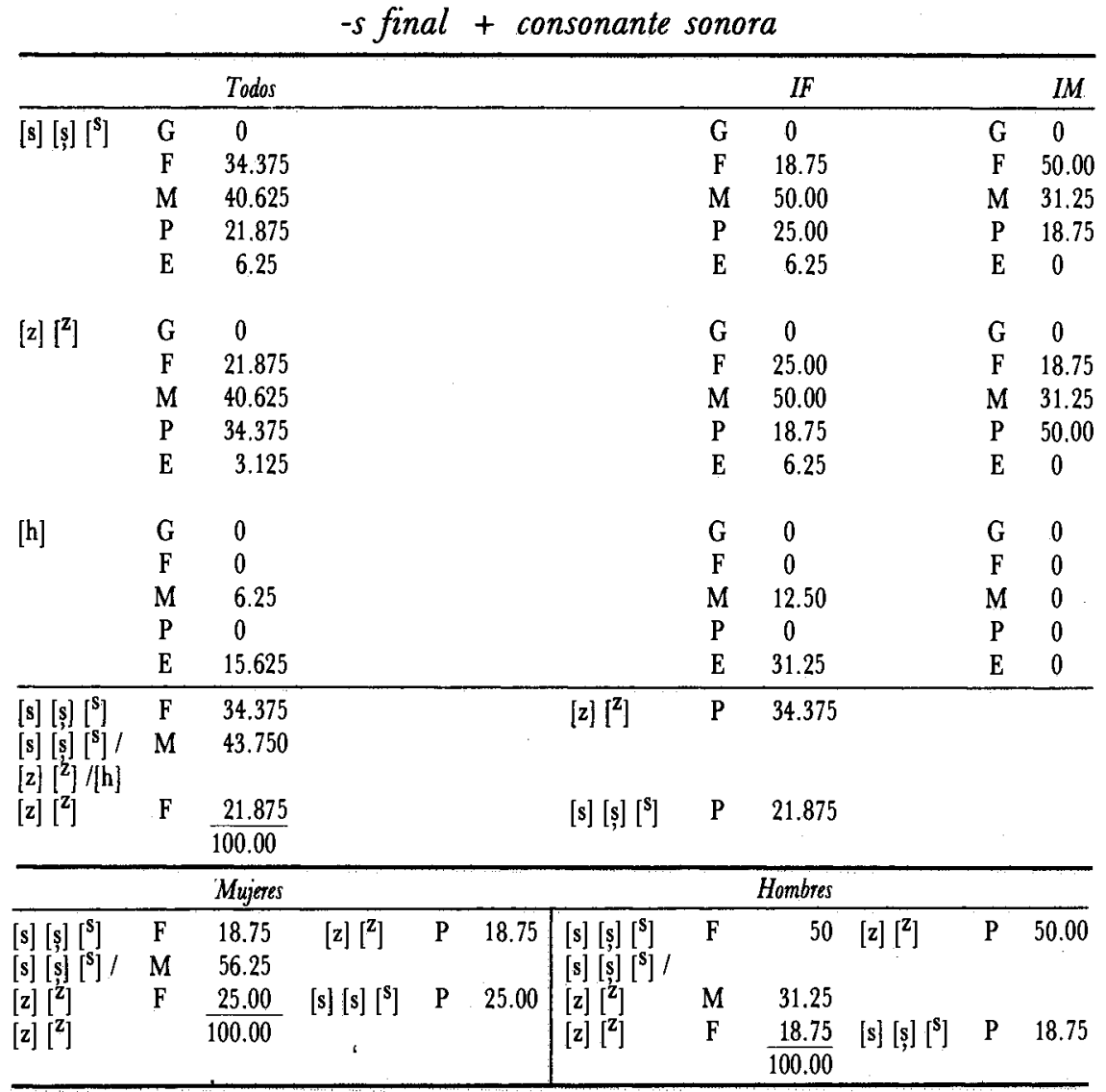

$/ l 1$

La articulación de /// como sonido alveolar lateral sonoro es general en el habla de la mayoría de la generación.joven estudiada. En un pequeño porcentaje el sonido es articulado con la lengua baja $[1 \perp]$. 
Ill

\begin{tabular}{ccccccccc}
\hline & Todos & & IF & & IM \\
\hline$[1]$ & $\mathrm{G}$ & 87.50 & $\mathrm{G}$ & 81.25 & & $\mathrm{G}$ & 93.75 \\
$\mathrm{~F}$ & 0 & $\mathrm{~F}$ & 0 & $\mathrm{~F}$ & 0 \\
$\mathrm{M}$ & 3.125 & $\mathrm{M}$ & 6.25 & $\mathrm{M}$ & 0 \\
$\mathrm{P}$ & 3.125 & $\mathrm{P}$ & 6.25 & & $\mathrm{P}$ & 0 \\
$\mathrm{E}$ & 0 & $\mathrm{E}$ & 0 & & $\mathrm{E}$ & 0
\end{tabular}

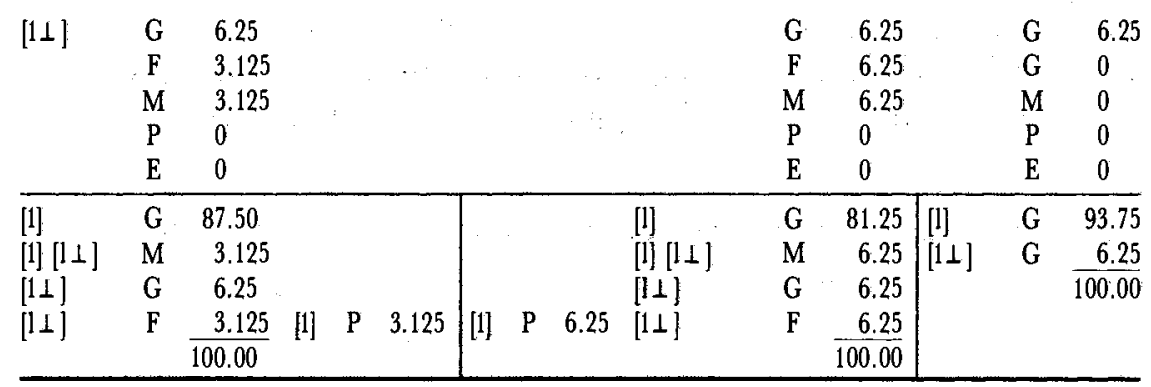

$|r|$

${ }^{-r}$ - El más frecuente de los alófonos articulados para -r- media es el sonido alveolar vibrante simple sonoro [r]. En segundo lugar está el alófono alveolar fricativo sonoro [*]. Hay mayor uso del sonido fricativo entre las mujeres y preferencia por el vibrante simple de parte de los hombres.

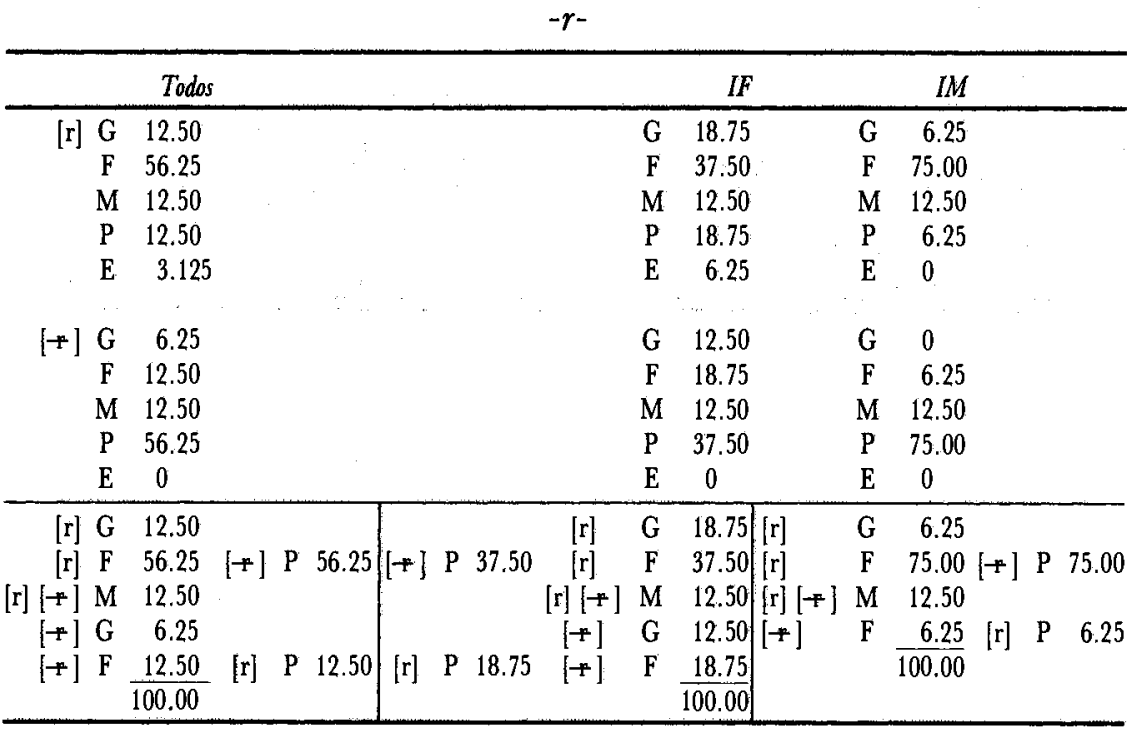


$t r, d r, g r$. En combinación con $/ \mathrm{t} /, / \mathrm{d} / \mathrm{y} / \mathrm{g} /$, el alófono usado generalmente por el $43.75 \%$ de los informantes es el vibrante simple sonoro con la lengua puesta contra los alveolos [r]. Tal uso se distribuye igualmente entre los dos sexos.

Ninguno de los informantes usa el fricativo [ ] generalmente en combinación con $t, d, g$. Sin embargo, casi la tercera parte de las mujeres lo hacen con frecuencia $(31.25 \%)$. Hay libre distribución entre [r] y [ ] en el habla de cuatro informantes masculinos $(25 \%)$. Se nota el predominio del sonido vibrante entre los varones, el cual es menos evidente entre las mujeres.

$t r-/ d r-/ g r-$

\begin{tabular}{|c|c|c|c|c|c|c|c|}
\hline \multicolumn{3}{|r|}{ Todos } & & \multicolumn{3}{|c|}{ IF } & $I M$ \\
\hline$[\mathrm{r}]$ & G & 43.75 & & G & 43.75 & G & 43.75 \\
\hline & $\mathrm{F}$ & 25.00 & & $\mathbf{F}$ & 25.00 & $\mathbf{F}$ & 25.00 \\
\hline & M & 15.625 & & M & 0 & M & 31.25 \\
\hline & $P$ & 15.625 & & $\mathbf{P}$ & 31.25 & $\mathrm{P}$ & 0 \\
\hline & E & 0 & & E & 0 & E & 0 \\
\hline$[+]$ & G & 0 & & G & 0 & G & 0 \\
\hline & $\mathbf{F}$ & 15.625 & & $\mathbf{F}$ & 31.25 & $\mathrm{~F}$ & 0 \\
\hline & M & 15.625 & & M & 0 & M & 31.25 \\
\hline & $P$ & 25.00 & & $\mathrm{P}$ & 25.00 & $P$ & 25.00 \\
\hline & E & 12.50 & & $\mathrm{E}$ & 12.50 & E & 12.50 \\
\hline$\left[\begin{array}{r}r \\
\mathbf{r}\end{array}\right]$ & G & 0 & & G & 0 & G & 0 \\
\hline & $\mathbf{F}$ & 0 & & $\mathbf{F}$ & 0 & $\mathbf{F}$ & 0 \\
\hline & M & 0 & & M & 0 & M & 0 \\
\hline & $\mathbf{P}$ & 0 & & $\mathbf{P}$ & 0 & $\mathbf{F}$ & 0 \\
\hline & $\mathrm{E}$ & 3.125 & & E & 6.25 & $\mathbf{F}$ & 0 \\
\hline$[\mathbf{r}]$ & G & 43.75 & {$[\mathbf{r}]$} & G & 43.75 & {$[\mathbf{r}]$} & 43.75 \\
\hline$[\mathrm{r}]$ & $\mathbf{F}$ & [+] P 25.00 & {$\left[\begin{array}{llll}+ & \text { P } & 25.00 & {[\mathrm{r}]}\end{array}\right.$} & $\mathbf{F}$ & 25.00 & {$[\mathrm{r}]$} & $25.00[-]$ P 25.00 \\
\hline$[\mathbf{r}][+]$ & & 15.625 & {$\left[\begin{array}{llll}r & P & 31.25 & {[-+}\end{array}\right]$} & & 31.25 & {$[\mathrm{r}][+] \mathrm{M}$} & 31.25 \\
\hline$[+]$ & $\mathrm{F}$ & $\frac{15.625}{100.00} \quad[r] \quad P \quad 15.625$ & & & 100.00 & & 100.00 \\
\hline
\end{tabular}


$-r$. La $r$ final es enunciada de tres maneras: $[r],[\leftarrow]$ y $[r]$, por la generación joven sonorense. En posición final de palabra, el alófono fricativo [-] predomina, pero con diferencias notables según el sexo del hablante. Casi la tercera parte de los varones usan [*] generalmente mientras que ninguna informante femenina articula $r$ final [ $\leftarrow$ ] como uso general. Sin embargo, el $75 \%$ de ellas la articulan [ + ] frecuentemente.

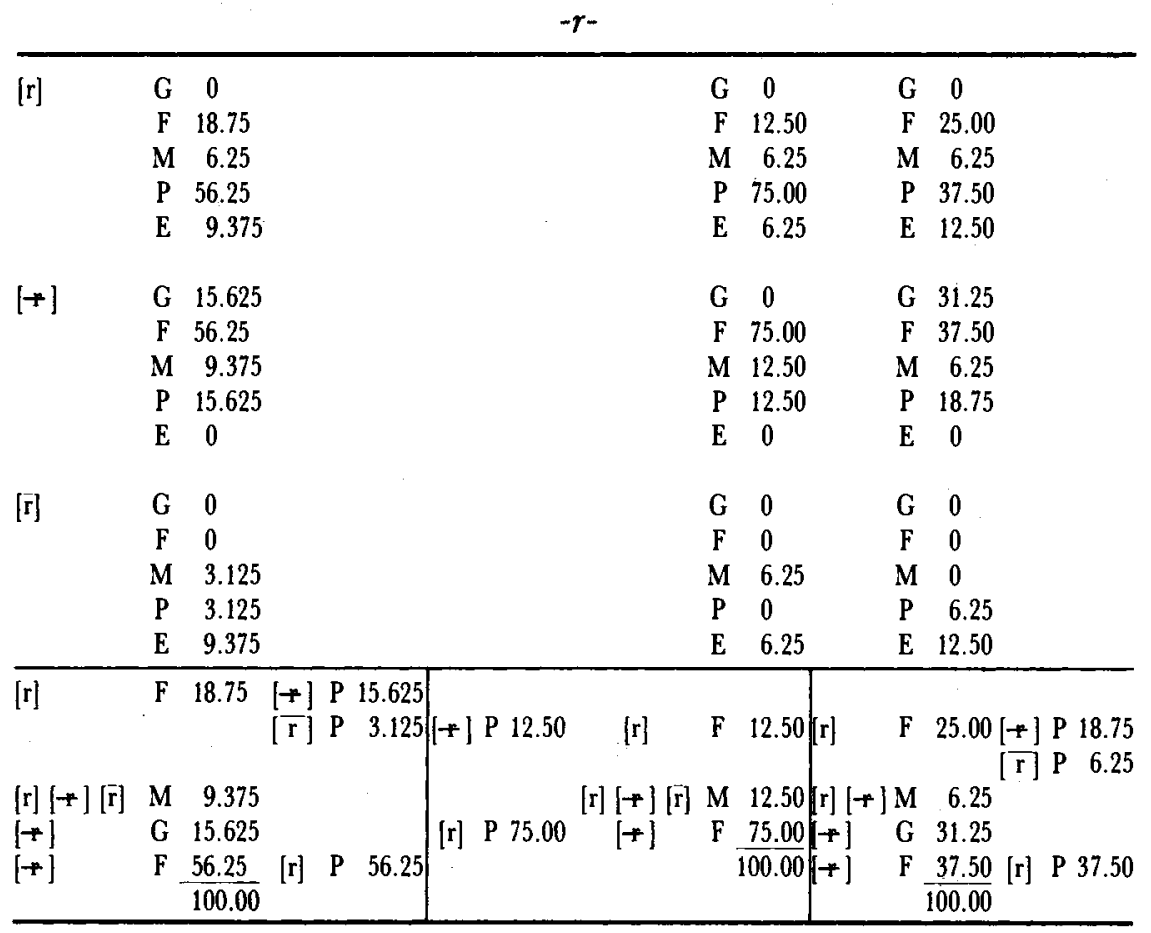


$\bar{r}^{-}$. Ejemplos de $r$ - inicial de palabra después de pausa o después de consonante ocurren en las grabaciones de 25 de los 32 informantes $(78.125 \%)$. El alófono de mayor frecuencia es el fricativo $[\bar{\Psi}]$. Más de la tercera parte de las jóvenes $(37.50 \%)$ usa el fricativo generalmente; menos de un quinto de los varones lo articula así (18.75\%). Lo que destaca en el caso de $r$ - es el plurimorfismo en el caso de la mayoría de los jóvenes de Sonora aquí estudiados.

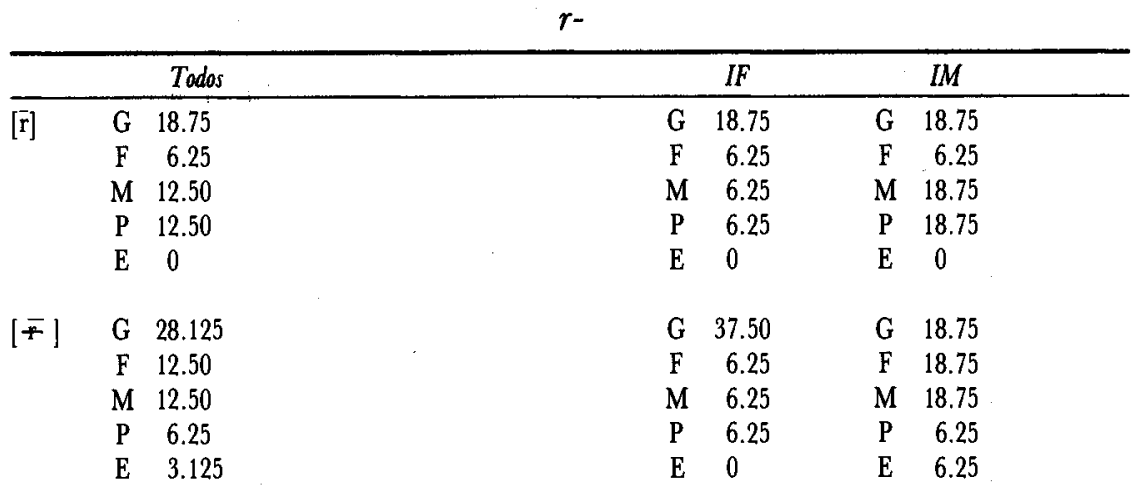

Sin ejemplos: 21.875 , F 25.00 y M 18.75

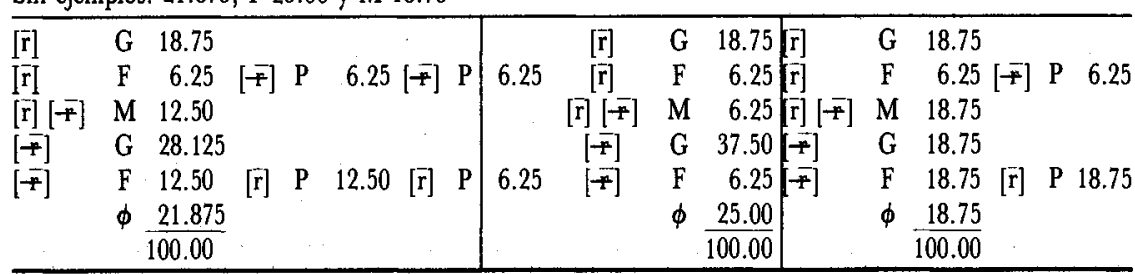


$-r r$-. La -rr-intervocálica se pronuncia generalmente como alveolar vibrante múltiple sonora $[\overline{\mathrm{r}}]$ por 10 de los 32 informantes $(31.25 \%)$. De este grupo, 7 son varones y 3 , mujeres. La articulación vibrante constituye la pronunciación general del $43.75 \%$ de los varones y del $18.75 \%$ de las mujeres. Sin embargo, cuando se revisan las cifras para uso general combinado con uso frecuente, la vibrante múltiple resulta la articulación favorecida tanto por parte de las mujeres (G 18.75\% , F $18.75 \%$ ) como de los hombres (G $43.75 \%$ y F $12.50 \%$ ).

Las diferencias de uso se alinean según el sexo del hablante: más informantes hombres que mujeres pronuncian -rr- generalmente como vibrante múltiple; tres veces más mujeres que hombres lo pronuncian como fricativo.

Se puede observar que la posición intervocálica -rr- provoca la articulación vibrante múltiple más que la posición inicial.

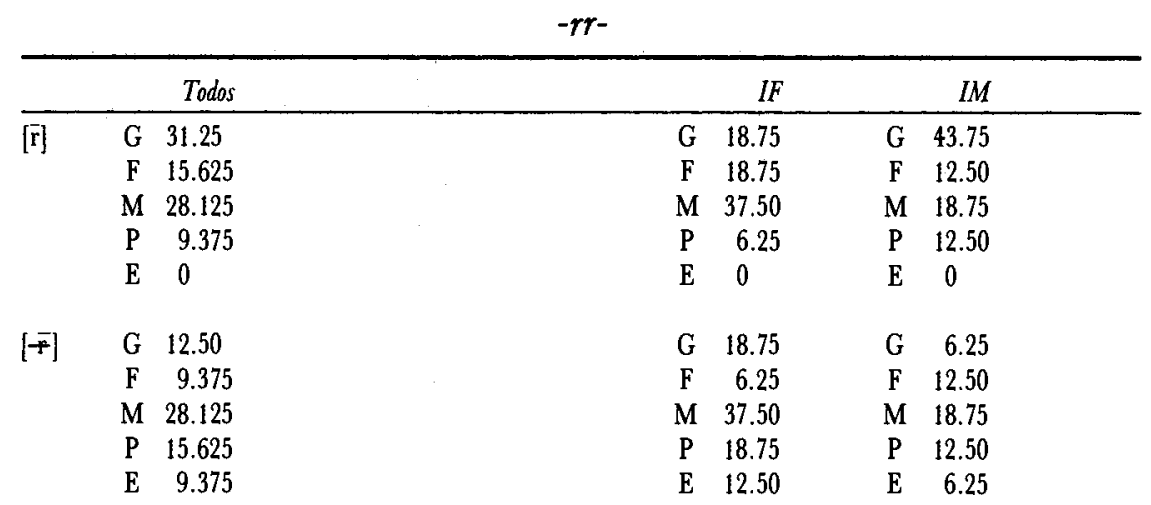

Sin ejemplos: 3.125 F 0 M 6.25

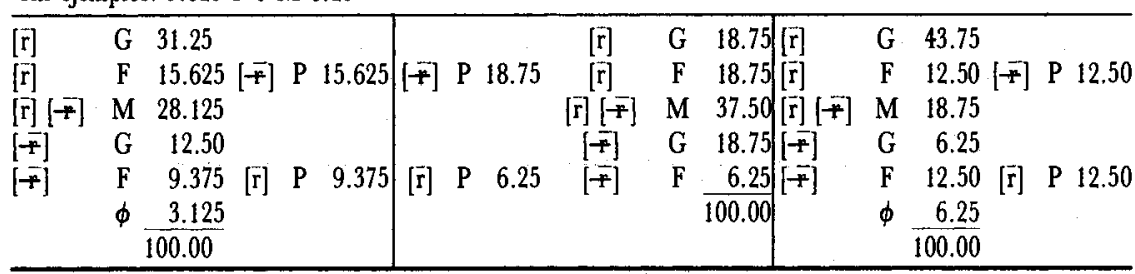


$/ m / i$

$\mathrm{La} / \mathrm{m} / \mathrm{se}$ articula como sonido bilabial nasal sonoro [m] en el habla de los informantes que constituyen el corpus lingüístico de este estudio. No se perciben variantes significativas.

$/ n / i$

$\mathrm{El}$ alófono alveolar nasal sonoro [n] es el que aparece generalmente en el habla de casi la mitad de los informantes (46.875\%). Los varones lo incorporan un poco más que las mujeres (M $50.00 \%$, F $43.75 \%$ ). La variante más notada después de [n] es la articulación con la lengua baja $[n \perp]$. Casi un tercio de las informantes articulan $n$ así generalmente (31.25\%), pero sólo un varón lo articula sin tensión $(6.25 \%)$ como regla general.

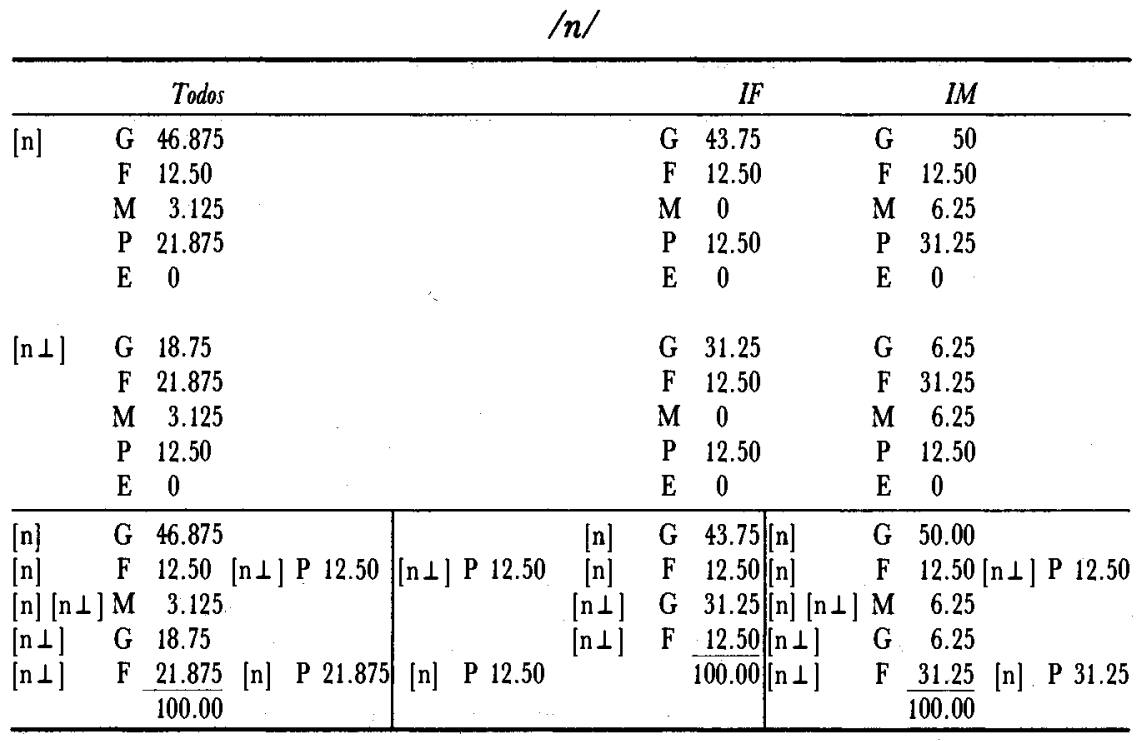


Entre los jóvenes examinados, la pronunciación característica de $/ \tilde{\mathbf{n}} /$ es el sonido palatal nasal sonoro relajado $[\tilde{\mathbf{n}} \perp]$. No aparece $/ \tilde{\mathbf{n}} /$ en casi una tercera parte de las conversaciones espontáneas grabadas, pero donde ocurre, suele ser relajado más que tenso.

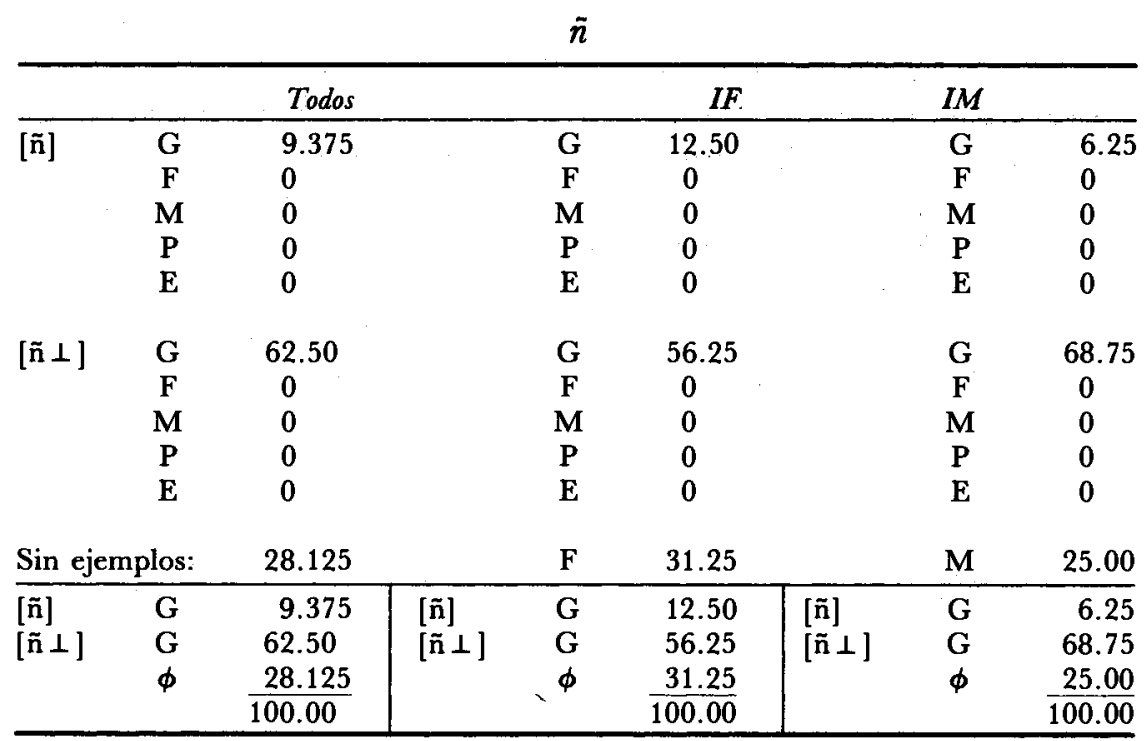


$\mid \hat{v}$

ch. Cuando $c h$ aparece sin influencia de sonido nasal anterior, dos terceras partes de los jóvenes estudiados la pronuncian generalmente como consonante palatal fricativa sonora [s] $(65.625 \%)$. Un pequeño número ( 2 mujeres) la articulan generalmente como palatal africada sonora [ $\hat{\mathrm{s}}$ ]. Al combinar las clasificaciones general y frecuente, la articulación fricativa se encuentra en el habla de $81.25 \%$ de los informantes, en contraste con el $12.50 \%$ cuya pronunciación general y frecuente es africada. Tales porcentajes permiten la categorización $[\hat{\mathrm{s}}$ ] de como alófono típico de la pronunciación juvenil de Sonora.

\begin{tabular}{|c|c|c|c|c|c|c|c|c|c|c|c|c|c|}
\hline & & & & & & & & IF & & & $I M$ & & \\
\hline \multirow{5}{*}[\begin{array}{c}{v}\\
{s}\end{array}]{} & $\mathrm{G}$ & 6.25 & & & & & & $\mathrm{G}$ & 12.50 & & $G$ & 0 & \\
\hline & $\mathrm{F}$ & 6.25 & & & & & & $\mathrm{~F}$ & 6.25 & & $\mathrm{~F}$ & 6.25 & \\
\hline & $\mathrm{M}$ & 6.25 & & & & & & M & 6.25 & & M & 6.25 & \\
\hline & $P$ & 15.625 & & & & & & $P$ & 12.50 & & $\mathrm{P}$ & 18.75 & \\
\hline & $\mathrm{E}$ & 9.375 & & & & & & $\mathrm{E}$ & 6.25 & & $\mathrm{E}$ & 12.50 & \\
\hline \multirow[t]{5}{*}[\begin{array}{c}{v}\\
{s}\end{array}]{} & $\mathrm{G}$ & 65.625 & & & & & & $G$ & 62.50 & & $\mathrm{G}$ & 68.75 & \\
\hline & $\mathrm{F}$ & 15.625 & & & & & & $\mathrm{~F}$ & 12.50 & & $\mathrm{~F}$ & 18.75 & \\
\hline & $\mathrm{M}$ & 6.25 & & & & & & $\mathrm{M}$ & 6.25 & & $\mathrm{M}$ & 6.25 & \\
\hline & $P$ & 6.25 & & & & & & $P$ & 6.25 & & $\mathrm{P}$ & 6.25 & \\
\hline & $\mathrm{E}$ & 3.125 & & & & & & $\mathrm{E}$ & 6.25 & & $\mathrm{E}$ & 0 & \\
\hline $\begin{array}{l}\hat{v} \\
{[s]}\end{array}$ & G & 6.25 & & & & & $\hat{v}$ & $\mathrm{G}$ & 12.50 & {$\left[\begin{array}{l}\hat{v} \\
{[s]}\end{array}\right.$} & $\mathrm{F}$ & $6.25[\mathrm{~s}] \mathrm{P}$ & P $\quad 6.25$ \\
\hline$[\hat{v}]$ & $\mathrm{F}$ & $6.25 \quad[\mathrm{v}]$ & $\mathbf{P}$ & 6.25 & {$\left[\begin{array}{l}\mathrm{v} \\
\mathrm{s}\end{array}\right] \mathrm{P}$} & 6.25 & {$[\hat{v}]$} & $\mathrm{F}$ & 6.25 & {$[\hat{v}]\left[\begin{array}{l}v \\
s\end{array}\right]$} & $\mathrm{M}$ & 6.25 & \\
\hline$[\hat{v}][\hat{s}]$ & M & 6.25 & & & 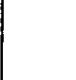 & & 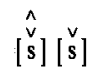 & $\mathrm{M}$ & 6.25 & {$[\stackrel{v}{s}]$} & $\mathrm{G}$ & 68.75 & \\
\hline$[\stackrel{v}{s}]$ & G & 65.625 & & & & & {$\left[\begin{array}{l}v \\
s\end{array}\right]$} & $G$ & 62.50 & {$\left[\begin{array}{l}v \\
s\end{array}\right]$} & $\mathrm{F}$ & $18.75\left[\begin{array}{l}\hat{v} \\
\text { s }\end{array}\right] \mathrm{P}$ & P 18.75 \\
\hline$[\stackrel{v}{s}]$ & $\mathrm{F}$ & $\frac{15.625}{100.00}[\hat{\mathrm{v}}]$ & $\mathrm{P}$ & 15.625 & {$[\hat{v}$} & 12.50 & {$\left[\begin{array}{l}v \\
s\end{array}\right]$} & $\mathrm{F}$ & $\frac{12.50}{100.00}$ & & & $\overline{100.00}$ & \\
\hline
\end{tabular}


Nasal $+c h$. El corpus para el ambiente de $c h$ precedida de nasal se reduce al habla de 13 informantes (4 hombres, 9 mujeres -el $40.685 \%$ del grupo). Más de la quinta parte de éstos articula el sonido generalmente como africado y el $15.625 \%$ lo hace fricativo. El fricativo es frecuente en el habla de otra informante $(3.125 \%)$.

Lo más notable en esa combinación es la diferencia de pronunciación entre ambos sexos: la articulación africada es general en las grabaciones de 4 informantes masculinos mientras 5 de las 9 mujeres generalmente producen la articulación fricativa.

Hay que tener en cuenta que la estadística para nasal $+c h$ no abarca todo el grupo estudiado, de manera que las cifras son indicaciones, más que conclusiones finales sobre la pronunciación de dicha combinación.

\section{nasal + ch}

\begin{tabular}{|c|c|c|c|c|c|}
\hline & & & $I F$ & & $I M$ \\
\hline$[\mathrm{s}]$ & 21.875 & G & 18.75 & G & 25.00 \\
\hline & 0 & $\mathrm{~F}$ & 0 & $\mathrm{~F}$ & 0 \\
\hline & 0 & $\mathbf{M}$ & 0 & M & 0 \\
\hline & 3.125 & $\mathbf{P}$ & 6.25 & $\mathbf{P}$ & 0 \\
\hline & 0 & $\mathbf{E}$ & 0 & E & 0 \\
\hline$[\mathbf{v}]$ & 15.625 & G & 31.25 & G & 0 \\
\hline & 3.125 & $\mathrm{~F}$ & 6.25 & $\mathrm{~F}$ & 0 \\
\hline & 0 & $\mathbf{M}$ & 0 & M & 0 \\
\hline . & 0 & $\mathbf{P}$ & 0 & $\mathbf{P}$ & 0 \\
\hline & 0 & E & 0 & E & 0 \\
\hline
\end{tabular}

Sin ejemplos: $59.375 \phi 43.75 \phi 75.00$

\begin{tabular}{|c|c|c|c|c|c|c|c|c|c|c|c|c|c|}
\hline$[\mathbf{v}$ ] & G & 21.875 & & & & & & [v] & G & 18.75 & {$[\mathrm{i}]$} & G & 25.00 \\
\hline$[\stackrel{v}{s}]$ & G & 15.625 & & & & & & [v s] & G & 31.25 & & $\phi$ & $\begin{array}{r}75.00 \\
10000\end{array}$ \\
\hline$[\mathrm{v}$ ] & $F$ & $3.125[\stackrel{v}{\mathrm{~s}}]$ & $P$ & 3.125 & [v s] & $\mathbf{P}$ & 6.25 & {$[\stackrel{v}{s}]$} & $F$ & 6.25 & & & \\
\hline & $\phi$ & $\frac{59.375}{100.00}$ & & & & & & & $\phi$ & $\frac{43.75}{100.00}$ & & & \\
\hline
\end{tabular}


$y$-, $l l$-. En posición inicial después de una pausa, $y$-, $l l$ - se articulan de dos maneras principales: [y] palatal rehilado sonoro y [y $\perp$ ] palatal rehilado sonoro con la lengua baja. Estos sonidos ocurren en las entrevistas de la mitad del grupo analizado (16 personas, $9 \mathrm{~F}$ y $7 \mathrm{M}, 28.125 \%$ y $21.857 \%$ respectivamente). La articulación [y] es general en el $18.75 \%$ de los informantes; [y $\perp$ ] es general en sólo el 3.125 por ciento.

La pronunciación africada se percibe poco en el habla femenil. Tal articulación no aparece en el habla masculina estudiada.

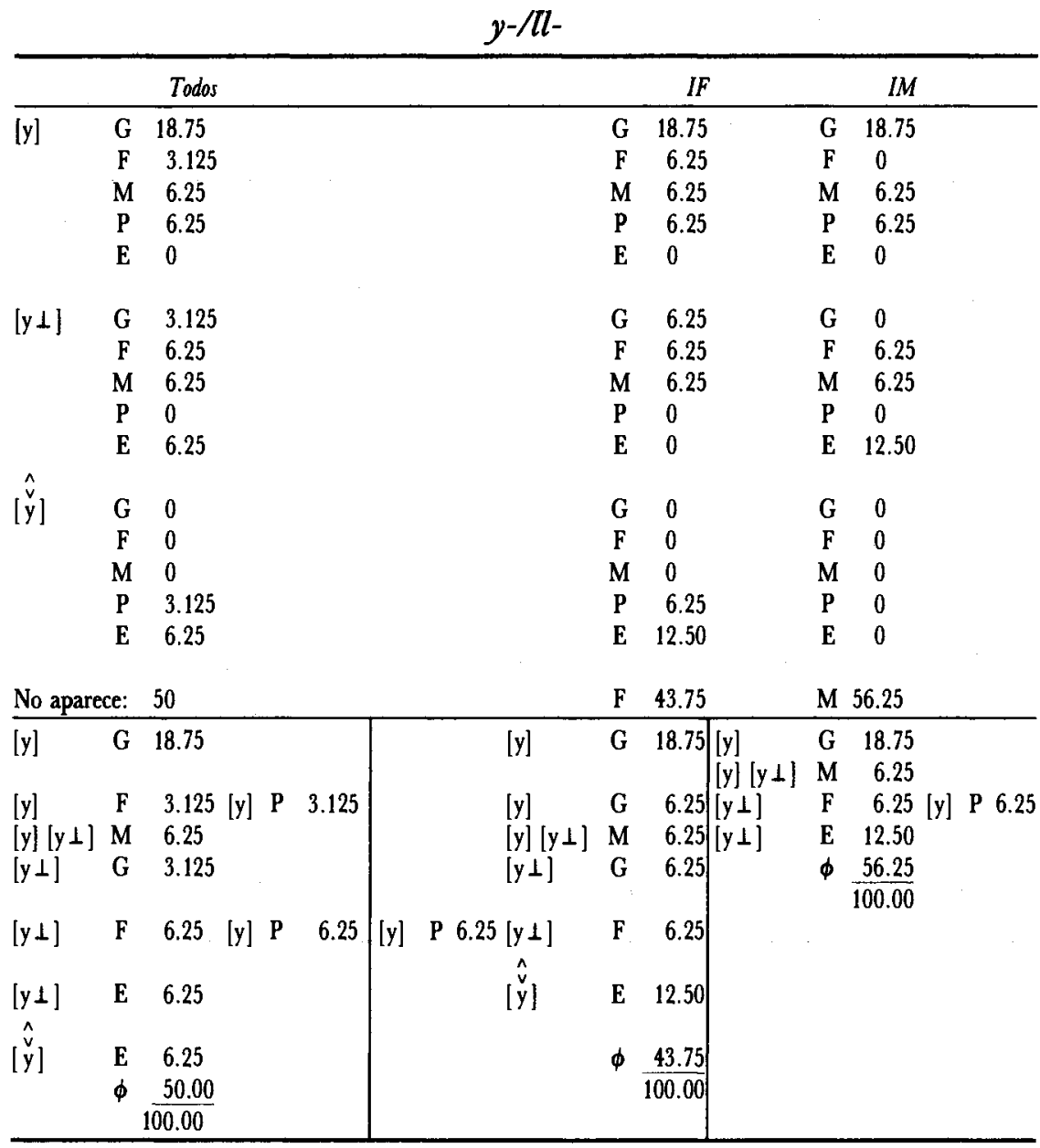


$-y-,-l l-$. El sonido rehilado sonoro [y] es el alófono encontrado en el habla de la cuarta parte del grupo juvenil $(25 \%)$. El sonido relajado, producido con relajamiento de la lengua en su contacto con el paladar $[\mathrm{y} \perp]$ es general en casi el $10 \%$ de los informantes entrevistados. Aunque hay menos diferencia en la categoría de uso frecuente que en la general, $[\mathrm{y}]$ se observa más que $[\mathrm{y} \perp] .[\hat{y}]$ sólo aparece esporádicamente en el habla de dos informantes $(6.25 \%)$.

$-y-,-l l-$

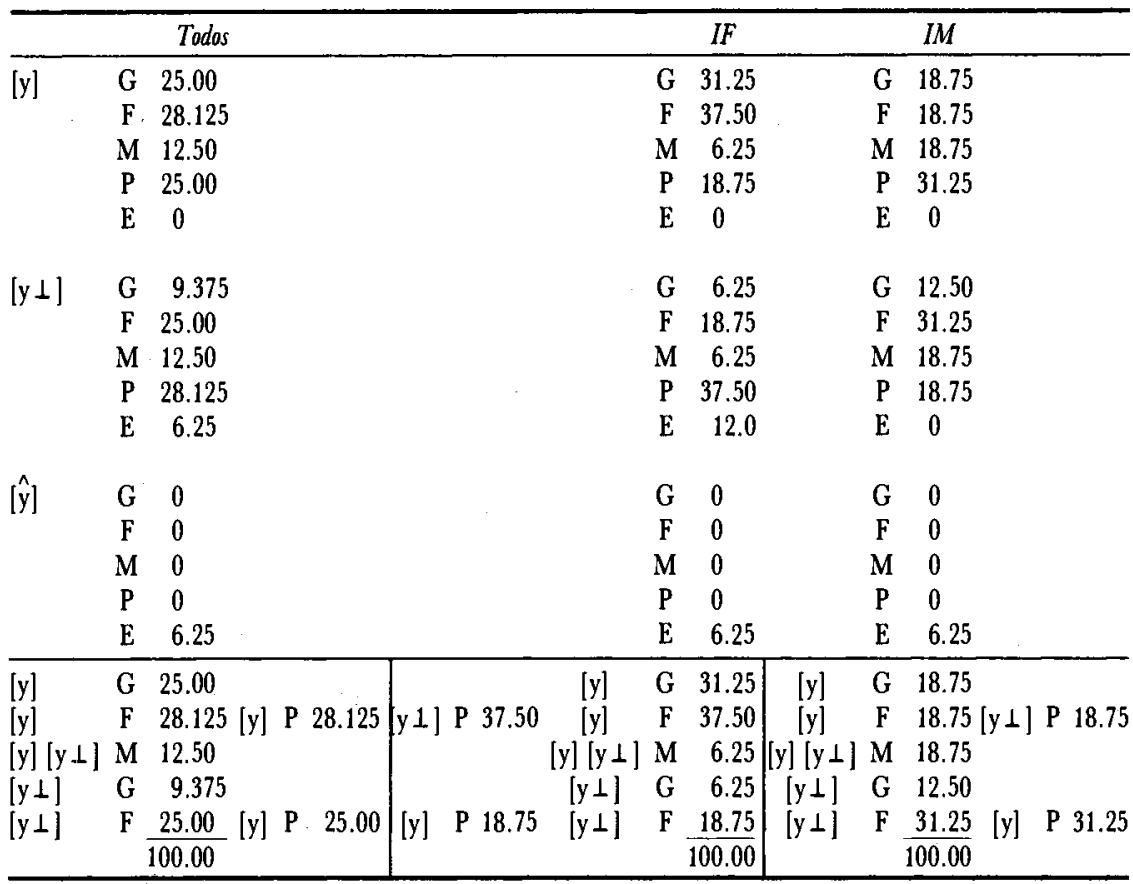


$/ w /$

El sonido bilabiovelar fricativo sonoro /w/ ocurre pocas veces en las conversaciones grabadas. Esas ocurrencias se encuentran en el habla de 8 informantes (2 F, $6 \mathrm{M}$ ). La articulación fuerte [w] se usa más que la pronunciación relajada $[\mathrm{w}]$. [w] es general en $18.75 \%$ de los informantes. Un varón articula el sonido generalmente relajado $\left[{ }^{\mathrm{W}}\right]$. Ninguna mujer lo hace.

En las entrevistas, el $75 \%$ de los jóvenes no tuvieron ocasión de articular el sonido /w/. Las cifras, por consiguiente, son parciales.

\begin{tabular}{ccccccc}
\multicolumn{8}{c}{ hua } \\
\hline & & Todos & & IF. & IM \\
\hline [w] & G & 18.75 & G & 12.0 & G & 25.00 \\
& F & 0 & F & 0 & F & 0 \\
& M & 3.125 & M & 0 & M & 6.25 \\
& P & 0 & P & 0 & P & 0 \\
& E & 0 & E & 0 & E & 0 \\
& & & & & & \\
{$\left[{ }^{\mathrm{w}}\right]$} & G & 3.125 & G & 0 & G & 6.25 \\
& F & 0 & F & 0 & F & 0 \\
& M & 3.125 & M & 0 & M & 6.125 \\
& P & 0 & P & 0 & P & 0 \\
& E & 0 & E & 0 & E & 0
\end{tabular}

\begin{tabular}{|c|c|c|c|c|c|c|c|c|}
\hline No apar & & 75 & & $\mathrm{~F}$ & 87.50 & & M & 62.50 \\
\hline $\begin{array}{l}{[\mathrm{w}]} \\
{[\mathrm{w}]} \\
{[\mathrm{w}]}\end{array}\left[^{\mathrm{w}}\right]$ & $\begin{array}{c}\mathrm{G} \\
\mathrm{M} \\
\mathrm{G} \\
\phi\end{array}$ & $\begin{array}{r}18.75 \\
3.125 \\
3.125 \\
75.00 \\
100.00\end{array}$ & {$[\mathbf{w}]$} & $\begin{array}{l}\mathrm{G} \\
\phi\end{array}$ & $\begin{array}{r}12.50 \\
87.50 \\
100.00\end{array}$ & $\begin{array}{c}{[w]} \\
{[w][w]} \\
{\left[{ }^{w}\right]} \\
\phi\end{array}$ & $\begin{array}{l}\text { G } \\
\mathbf{M} \\
\mathbf{G}\end{array}$ & $\begin{array}{r}25.00 \\
6.25 \\
6.25 \\
62.50 \\
100.00\end{array}$ \\
\hline
\end{tabular}


$|x|$

$j, g e, g i$. La mitad de los jóvenes estudiados articulan $j, g e, g i$ generalmente como consonante glotal fricativa sorda [h]. Ninguno de los informantes analizados pronuncia el sonido como velar $[\mathrm{x}]$ generalmente y de los 32 informantes, sólo una mujer lo hace frecuentemente. El 40.625\% incorpora el glotal [h] frecuentemente. Estas cifras muestran la preferencia por el alófono glotal de parte de la generación joven sonorense por encima del alófono velar.

$j, g e, g i$

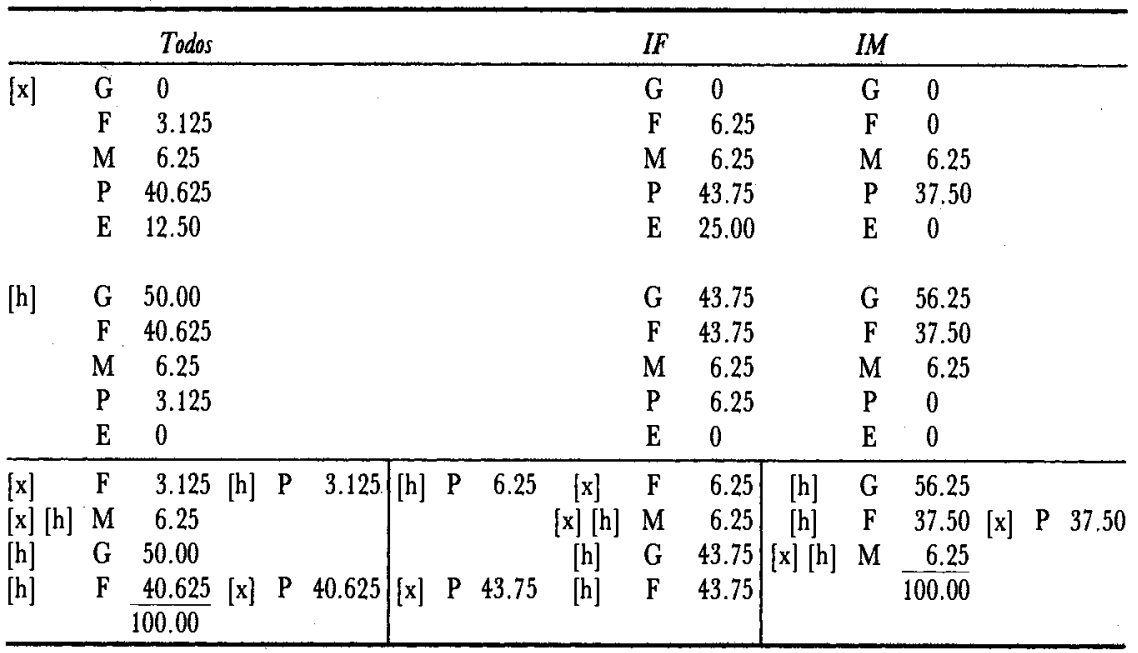




\section{La letra $x$}

El grafema $x$ aparece 36 veces en las conversaciones grabadas. Únicamente 14 de los 32 informantes recurrieron a palabras escritas con $x(10 \mathrm{~F}, 4 \mathrm{M})$. La combinación consonántica se articula de cuatro maneras: [ks] (o $\left.\left[\mathrm{ks}_{\mathbf{s}}\right]\right),\left[\mathrm{k}_{\mathrm{s}}\right]$ con la lengua en una posición baja al principio de la articulación [s] (o [s]) y con un sonido dental y oclusivo entre los dos sonidos $\left[\mathrm{k}^{\mathrm{t}} \mathrm{s}\right]$.

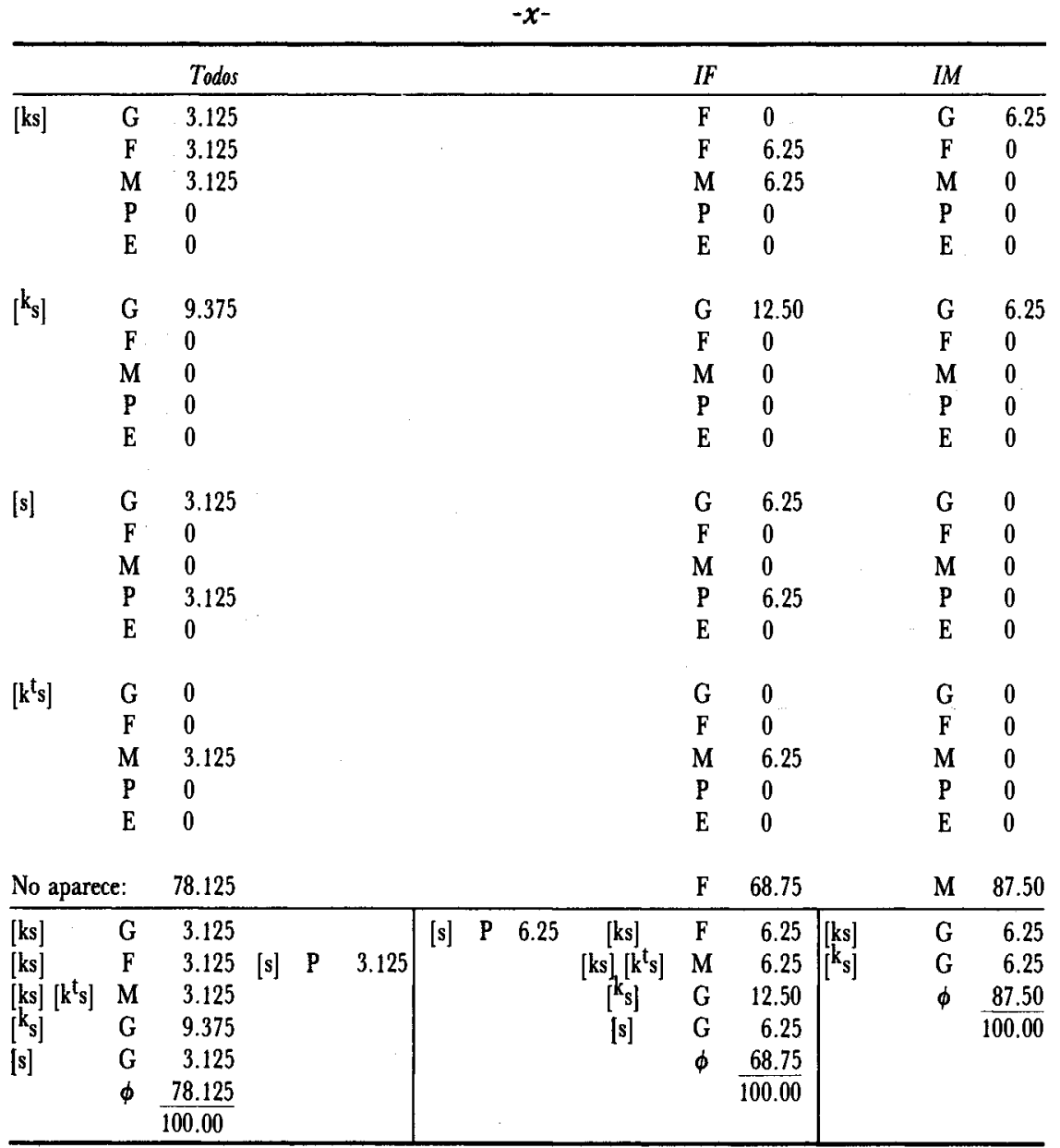


$x+$ consonante. La combinación $x+$ consonante aparece en sólo el $31.25 \%$ de los informantes grabados (F 10, M 2). La articulación $[\mathrm{ks}]$ (o [ks]) es general en la mayor parte de esos informantes; [ $\left.\mathrm{k}_{\mathrm{s}}\right]$ sigue en frecuencia y la reducción a [s] se encuentra en un informante masculino. Tres personas $(9.375 \%)$ alternan entre $[\mathrm{ks}]$ y $\left[\mathrm{k}_{\mathrm{s}}\right.$. El número de ejemplos $x+$ consonante no es suficiente para dar más indicaciones de su articulación por la generación joven sonorense.

$$
x+\text { cons. }
$$

\begin{tabular}{|c|c|c|c|c|c|c|c|c|}
\hline & & Todos & & IF & & & $I M$ & \\
\hline$[\mathrm{ks}] / / \mathrm{ks}]$ & $\begin{array}{l}\text { G } \\
F \\
M \\
P \\
E\end{array}$ & $\begin{array}{l}9.375 \\
3.125 \\
9.375 \\
0 \\
0\end{array}$ & & $\begin{array}{l}\mathrm{G} \\
\mathrm{F} \\
\mathrm{M} \\
\mathrm{P} \\
\mathrm{E}\end{array}$ & $\begin{array}{c}12.50 \\
6.25 \\
18.75 \\
0 \\
0\end{array}$ & & $\begin{array}{l}\mathrm{G} \\
\mathrm{F} \\
\mathrm{M} \\
\mathrm{P} \\
\mathrm{E}\end{array}$ & $\begin{array}{l}6.25 \\
0 \\
0 \\
0 \\
0\end{array}$ \\
\hline$\left[\mathrm{k}_{\mathrm{s}}\right]$ & $\begin{array}{l}\text { G } \\
\text { F } \\
N \\
\text { P } \\
\text { E }\end{array}$ & $\begin{array}{l}6.25 \\
0 \\
9.375 \\
3.125 \\
0\end{array}$ & & $\begin{array}{l}\text { G } \\
\text { F } \\
M \\
P \\
\text { E }\end{array}$ & $\begin{array}{c}12.50 \\
0 \\
18.75 \\
6.25 \\
0\end{array}$ & & $\begin{array}{l}\text { G } \\
\text { F } \\
M \\
\text { P } \\
\text { E }\end{array}$ & $\begin{array}{l}0 \\
0 \\
0 \\
0 \\
0\end{array}$ \\
\hline [s] & $\begin{array}{l}\text { G } \\
F \\
M \\
P \\
E\end{array}$ & $\begin{array}{l}3.125 \\
0 \\
0 \\
0 \\
0\end{array}$ & & $\begin{array}{l}\mathrm{G} \\
\mathrm{F} \\
\mathrm{M} \\
\mathrm{P} \\
\mathrm{E}\end{array}$ & $\begin{array}{l}0 \\
0 \\
0 \\
0 \\
0\end{array}$ & & $\begin{array}{l}\mathrm{G} \\
\mathrm{F} \\
\mathrm{M} \\
\mathrm{P} \\
\mathrm{E}\end{array}$ & $\begin{array}{l}6.25 \\
0 \\
0 \\
0 \\
0\end{array}$ \\
\hline No aparece: & & 68.75 & & F & 50.00 & & M & 87.50 \\
\hline $\begin{array}{l}{[\mathrm{ks}][\mathrm{ks}]} \\
{[\mathrm{ks}][\mathrm{ks}]} \\
{[\mathrm{ks}][\mathrm{ks}]\left[\mathrm{k}_{\mathrm{s}}\right]} \\
{\left[\mathrm{k}_{\mathrm{s}}^{\prime}\right]} \\
{[\mathrm{s}]}\end{array}$ & $\begin{array}{l}\text { G } \\
\text { F } \\
M \\
\text { G } \\
\text { G } \\
\phi\end{array}$ & $\begin{array}{l}9.375 \\
3.125\left[\mathrm{k}_{\mathrm{s}}\right] \mathrm{P} \\
9.375 \\
6.25 \\
3.125 \\
68.75 \\
100.00\end{array}$ & 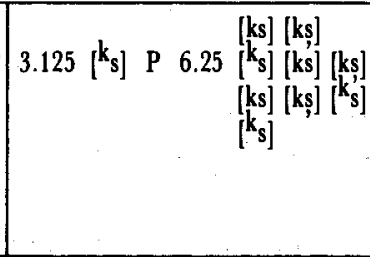 & $\begin{array}{l}G \\
F \\
M \\
G \\
\phi\end{array}$ & $\begin{array}{r}12.50 \\
6.25 \\
18.75 \\
12.50 \\
50.00 \\
100.00\end{array}$ & $\begin{array}{l}{[\mathbf{k s}][\mathbf{k s}]} \\
{[\mathbf{s}]} \\
.\end{array}$ & $\begin{array}{l}G \\
\phi\end{array}$ & $\begin{array}{r}6.25 \\
87.50 \\
100.00\end{array}$ \\
\hline
\end{tabular}


$-x$. Hay dos casos de $-x$ final de palabra en el material grabado: una informante (núm. 2) habla de un colegio particular, "Colegio Lux" [ $\mathrm{k}_{\mathrm{s}}$ ] y otra (núm. 12) pronuncia el nombre de una fiesta religiosa, "Corpus Christi"' [kór-puks] de una manera que se puede considerar ultracorrecta.

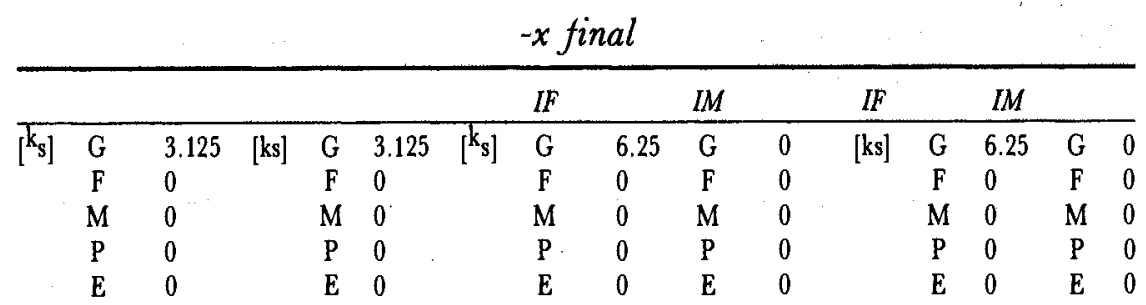

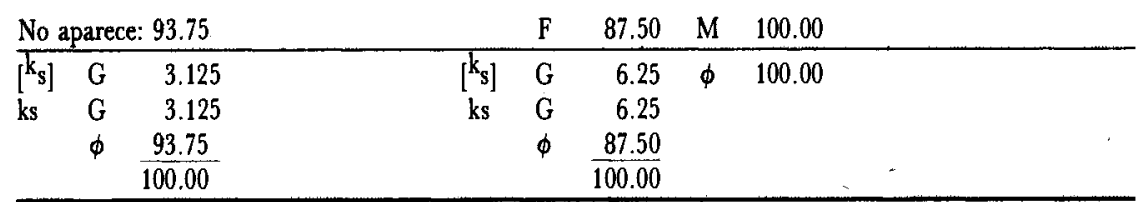

Modificaciones fONÉticas

\section{Ensordecimiento}

La articulación de sonidos sonoros sin vibración de las cuerdas vocales -el ensordecimiento- es un fenómeno que se encuentra en el habla espontánea de la generación joven sonorense. El ensordecimiento ocurre en tres ambientes principales: (1) vocal átona final, (2) consonante sonora anterior junto con la vocal átona final; y (3) vocal final átona más -s.

El ensordecimiento se da más en el habla femenil que en la masculina.

La cuarta parte de las mujeres observadas ensordecen la vocal átona final como regla general. Hay que señalar que el ensordecimiento es frecuente en el habla de la mayoría de las informantes femeninas. Casi dos tercios de ellas ensordecen la vocal átona final frecuentemente y la mitad de ellas ensordecen frecuentemente la vocal átona que es final ante $-s(50 \%)$. Esas cifras contrastan con el uso masculino: en sólo tres hablantes, es general el ensordecimiento de vocal átona final.

Según las grabaciones hechas a 32 jóvenes sonorenses, se puede observar que el ensordecimiento de vocal al final de la palabra es característico del habla femenil. 


\section{Ensordecimiento}

\begin{tabular}{lccccr}
\hline \multicolumn{5}{c}{ vocal átona final } \\
\hline $\mathrm{G}$ & 15.625 & $\mathrm{G}$ & 25.00 & $\mathrm{G}$ & 6.25 \\
$\mathrm{~F}$ & 46.875 & $\mathrm{~F}$ & 62.50 & $\mathrm{~F}$ & 31.25 \\
$\mathrm{M}$ & 21.875 & $\mathrm{M}$ & 6.25 & $\mathrm{M}$ & 37.50 \\
$\mathrm{P}$ & 9.375 & $\mathrm{P}$ & 6.25 & $\mathrm{P}$ & 12.50 \\
$\mathrm{E}$ & 0 & $\mathrm{E}$ & 0 & $\mathrm{E}$ & 0
\end{tabular}

\begin{tabular}{lccrcr} 
No aparece: & 6.25 & F & 0 & M & 12.50 \\
\hline G & 15.625 & G & 25.00 & G & 6.25 \\
F & 46.857 & G & 62.50 & F & 31.25 \\
M & 21.875 & M & 6.25 & M & 37.50 \\
P & 9.375 & $\mathrm{P}$ & 6.25 & P & 12.50 \\
$\phi$ & 6.25 & & 100.00 & $\phi$ & 12.50 \\
\hline
\end{tabular}

\begin{tabular}{lccrcc}
\hline \multicolumn{7}{c}{ consonante } & sonora + vocal átona final \\
\hline $\mathrm{G}$ & 3.125 & $\mathrm{G}$ & 6.25 & $\mathrm{G}$ & 0 \\
$\mathrm{~F}$ & 37.50 & $\mathrm{~F}$ & 68.75 & $\mathrm{~F}$ & 6.25 \\
$\mathrm{M}$ & 12.50 & $\mathrm{M}$ & 6.25 & $\mathrm{M}$ & 18.75 \\
$\mathrm{P}$ & 18.75 & $\mathrm{P}$ & 12.50 & $\mathrm{P}$ & 25.00 \\
$\mathrm{E}$ & 0 & $\mathrm{E}$ & 0 & $\mathrm{E}$ & 0
\end{tabular}

\begin{tabular}{|c|c|c|c|c|c|}
\hline No aparece: & 28.125 & $\mathbf{F}$ & 6.25 & $\mathbf{M}$ & 50.00 \\
\hline G & 3.125 & G & 6.25 & G & 6.25 \\
\hline F & 37.50 & $\mathbf{F}$ & 68.75 & $\mathbf{M}$ & 18.75 \\
\hline $\mathbf{M}$ & 12.50 & $\mathrm{M}$ & 6.25 & $\mathbf{P}$ & 25.00 \\
\hline $\mathbf{P}$ & 18.75 & $\mathbf{P}$ & 12.50 & $\phi$ & 50.00 \\
\hline$\phi$ & $\frac{28.125}{100.00}$ & $\phi$ & $\frac{6.25}{100.00}$ & & 100.00 \\
\hline \multicolumn{6}{|c|}{ vocal átona final +- s final } \\
\hline G & 3.125 & $G$ & 0 & G & 6.25 \\
\hline F & 28.125 & $\mathbf{F}$ & 50.00 & $\mathrm{~F}$ & 6.25 \\
\hline $\mathbf{M}$ & 6.25 & M & 0 & $\mathbf{M}$ & 12.50 \\
\hline $\mathbf{P}$ & 21.875 & $\mathbf{P}$ & 12.50 & $\mathbf{P}$ & 31.25 \\
\hline $\mathbf{E}$ & 0 & E & 0 & $\mathbf{E}$ & 0 \\
\hline No aparece: & 40.625 & F & 37.50 & $\mathbf{M}$ & 43.75 \\
\hline $\mathrm{G}$ & 3.125 & F & 50 & G & 6.25 \\
\hline $\mathbf{F}$ & 28.125 & $\mathbf{P}$ & 12.50 & $\mathbf{F}$ & 6.25 \\
\hline M & 6.25 & $\phi$ & 37.50 & $\mathbf{M}$ & 12.50 \\
\hline $\mathbf{P}$ & 21.875 & & $\overline{100.00}$ & $\mathbf{P}$ & 31.25 \\
\hline \multirow[t]{2}{*}{$\phi$} & 40.625 & & & $\phi$ & 43.75 \\
\hline & 100.00 & & & & 100.00 \\
\hline
\end{tabular}


Los alófonos principales del español mexicano se encuentran tanto en el habla de las 16 mujeres como en la de los 16 hombres. Al mismo tiempo, un análisis detallado de los sonidos observados en conversaciones espontáneas indica el predominio de ciertas variantes según el sexo de la persona que habla.

Se ha observado que las informantes femeninas tienen una mayor tendencia que los informantes masculinos a lo siguiente:

1. Usar libre variación entre los alófonos.

2. Elevar las vocales finales $-o[\mathrm{o}]$ y $-e[\mathrm{e}]$.

3. Articular $/ \mathrm{s} /$ como sonido dental [s].

4. Pronunciar $/ \mathrm{r} /$ como sonido fricativo [ $\leftarrow$ ].

5. Articular $c h$ y $y, l l$, como sonidos africados $[\hat{\mathrm{s}}]$ y $[\hat{y}]$ respectivamente.

6. Bajar la lengua al enunciar $k, l, n$ y $y$.

7. Ensordecer las vocales finales.

Los informantes masculinos tienen mayor tendencia que las informantes femeninas a lo siguiente:

1. Velarizar $a$ [a] $]$.

2. Articular /s/ como sonido alveolar [s].

3. Palatalizar si + vocal $[$ s $]$

4. Debilitar o perder el elemento oclusivo de [ $\hat{\text { š }}$ y articular ch como sonido fricativo [ $\stackrel{\mathrm{s}}{\mathrm{s}}$.

5. Pronunciar $/ \mathrm{r} / \mathrm{y} / \overline{\mathbf{r}} /$ como sonidos alveolares $[\mathrm{r}]$ y $[\overline{\mathbf{r}}]$.

A partir de estas tendencias se ve que el habla masculina sonorense tiende a velarizar y a palatalizar los sonidos mientras que el habla femenina tiende a elevar, a dentalizar, a hacer fricativos y a ensordecer los sonidos.

Puesto que las mujeres varían el uso de los alófonos, por ejemplo, entre $[\mathrm{s}]$ y $[s],[\mathrm{r}]$ y $[\mathbf{\leftarrow}],[\overline{\mathrm{r}}]$ y $[\overline{\mathbf{x}}],[\mathrm{x}]$ y $[\mathrm{h}]$, se puede concluir que las jóvenes sonorenses tienden a incorporar mayor variedad alofónica que sus contrapartidas masculinas. En otras palabras, hay más variación libre, mayor poliformismo en el habla femenil que en la masculina.

DOLORES Brown Universidad de Arizona 